\title{
GALAXY EVOLUTION IN OVERDENSE ENVIRONMENTS AT HIGH REDSHIFT: PASSIVE EARLY-TYPE GALAXIES IN A CLUSTER AT $z \sim 2$
}

\author{
V. Strazzullo ${ }^{1}$, R. Gobat ${ }^{1}$, E. Daddi ${ }^{1}$, M. Onodera ${ }^{2}$, M. Carollo $^{2}$, M. Dickinson ${ }^{3}$, A. Renzini ${ }^{4}$, \\ N. Arimoto 5,6 , A. Cimatti ${ }^{7}$, A. Finoguenov ${ }^{8}$, and R.-R. Chary $^{9}$ \\ ${ }^{1}$ CEA Saclay, Orme des Merisiers, F-91191 Gif sur Yvette, France \\ 2 Institute for Astronomy, ETH Zürich Wolfgang-Pauli-strasse 27, CH-8093 Zürich, Switzerland \\ ${ }^{3}$ National Optical Astronomy Observatory, P.O. Box 26732, Tucson, AZ 85726, USA \\ ${ }^{4}$ INAF, Osservatorio Astronomico di Padova Vicolo dell Osservatorio 5, I-35122 Padova, Italy \\ 5 Subaru Telescope, 650 North Aohoku Place, Hilo, HI 96720, USA \\ ${ }^{6}$ Graduate University for Advanced Studies, 2-21-1 Osawa, Mitaka, Tokyo 181-8588, Japan \\ ${ }^{7}$ Università di Bologna, Dipartimento di Astronomia, via Ranzani 1, I-40127 Bologna, Italy \\ ${ }^{8}$ Department of Physics, University of Helsinki, Gustaf Hällströmin katu 2a, FI-00014 Helsinki, Finland \\ ${ }^{9}$ Division of Physics, Mathematics and Astronomy, California Institute of Technology, Pasadena, CA 91125, USA \\ Received 2012 December 20; accepted 2013 May 13; published 2013 July 15
}

\begin{abstract}
We present a study of galaxy populations in the central region of the IRAC-selected, X-ray-detected galaxy cluster Cl J1449+0856 at $z=2$. Based on a sample of spectroscopic and photometric cluster members, we investigate stellar populations and the morphological structure of cluster galaxies over an area of $\sim 0.7 \mathrm{Mpc}^{2}$ around the cluster core. The cluster stands out as a clear overdensity both in redshift space and in the spatial distribution of galaxies close to the center of the extended X-ray emission. The cluster core region $(r<200 \mathrm{kpc})$ shows a clearly enhanced passive fraction with respect to field levels. However, together with a population of massive, passive galaxies mostly with early-type morphologies, the cluster core also hosts massive, actively star-forming, often highly dust reddened sources. Close to the cluster center, a multi-component system of passive and star-forming galaxies could represent the future brightest cluster galaxy still forming. We observe a clear correlation between passive stellar populations and an early-type morphology, in agreement with field studies at similar redshift. Passive early-type galaxies in this cluster are typically a factor of 2-3 smaller than similarly massive early types at $z \sim 0$. On the other hand, these same objects are on average larger by a factor of $\sim 2$ than field early-types at similar redshift, lending support to recent claims of an accelerated structural evolution in high-redshift dense environments. These results point toward the early formation of a population of massive galaxies, already evolved both in their structure and stellar populations, coexisting with still actively forming massive galaxies in the central regions of young clusters 10 billion years ago.
\end{abstract}

Key words: galaxies: clusters: individual (Cl J1449+0856) - galaxies: evolution - galaxies: high-redshift galaxies: stellar content - galaxies: structure

Online-only material: color figures

\section{INTRODUCTION}

In the nearby universe, and at least out to $z \sim 1$, overdense environments and specifically galaxy cluster cores are invariably found to preferentially host galaxy populations dominated by massive, passive early-type galaxies (e.g., among many others, Dressler 1980; Postman et al. 2005; Baldry et al. 2006; van der Wel et al. 2007; Patel et al. 2009; Rosati et al. 2009; Peng et al. 2010b; Wetzel et al. 2012). The way these galaxies form and evolve has been a longstanding matter of debate: their star formation histories, including differences with respect to field galaxies, have been investigated by high-redshift observations (e.g., van Dokkum \& van der Marel 2007; Gobat et al. 2008; Mei et al. 2009; Rettura et al. 2010) as well as fossil-record studies (e.g., Thomas et al. 2005, 2010). Most studies agree on the early formation of a population of massive cluster early types, with their stars formed at high redshift $(z \sim 2$ or beyond) and their mass largely assembled before $z \sim 1$ (e.g., De Propris et al. 2007; Lidman et al. 2008; Andreon 2008; Mancone et al. 2010; Strazzullo et al. 2010).

While detailed studies of cluster galaxy populations are relatively common up to $z \sim 1$, they become increasingly rarer at higher redshifts, and in particular beyond $z \sim 1.5$ due to observational challenges both in reliably identifying clusters and in accurately determining the properties of their galaxies. Nonetheless, the $z>1.5$ range is a crucial time to study massive cluster galaxies close to their main formation epoch. Indeed, recent observations of $z \gtrsim 1.5$ clusters started to show that massive galaxy populations are often still actively forming, even in the cluster core (e.g., Tran et al. 2010; Hilton et al. 2010; Hayashi et al. 2010, 2011; Santos et al. 2011; Fassbender et al. 2011b).

From a theoretical point of view, current models, while invoking an early formation for the stars ending up in massive early-type galaxies today, maintain their hierarchical nature in predicting the late assembly of stellar mass from smaller, mostly passive progenitors (e.g., De Lucia et al. 2006; Johansson et al. 2012). The relevance of such merging events, as well as other processes (e.g., active galactic nucleus (AGN) or stellar feedback), possibly affecting both the star formation history (SFH) and the galaxy structure in the evolutionary path of these systems, may be probed by the (albeit biased and complicated) comparison of cluster galaxy samples at different redshifts.

Reaching cosmic epochs when massive cluster galaxies are still forming is thus fundamental in order to directly observe the formation of the bulk of the stars, the way stellar mass is 
assembled, and morphological evolution, which together lead to massive early-types dominating cluster cores at later times.

Ideally, this kind of investigation would be carried out in clusters that are not pre-selected based on the characteristics of their galaxy populations, but are rather identified based on their mass or overdensity. At this redshift, and with current facilities, $\mathrm{X}$-ray selection becomes very challenging for the identification of moderately massive systems representative of the progenitors of typical lower-redshift clusters. On the other hand, "IRAC-selected" clusters identified based on overdensities of stellar-mass-limited galaxy samples (Eisenhardt et al. 2008; Papovich 2008), ideally with a posteriori detection of (generally faint) X-ray emission, offer a suitable alternative beyond $z \sim 1.5$.

In this work, we study galaxy populations in the IRAC-selected and X-ray-detected cluster Cl J1449+0856 at $z=2$ (R.A. $=14^{\mathrm{h}} 49^{\mathrm{m}} 14^{\mathrm{s}}$, decl. $=8^{\circ} 56^{\prime} 21^{\prime \prime}$; Gobat et al. 2011, 2013). This is among the most distant spectroscopicallyconfirmed galaxy clusters discovered so far, and the most distant with detected X-ray emission. The first spectroscopic investigation with VLT/VIMOS and FORS2 spectroscopy over a wide field around the cluster found a peak in the redshift distribution of star-forming galaxies at $z \sim 2.07$ (Gobat et al. 2011).

Subsequent follow-up on the cluster center with slitless $H S T /$ WFC3 spectroscopy unveiled a much stronger peak in the redshift distribution at $z=2$, which is the most prominent peak in the area of the galaxy overdensity. This peak contains about 20 spectroscopically-identified cluster members to date, including massive, passive red galaxies in the cluster core (Gobat et al. 2013). Cl J1449+0856 is thus now spectroscopically confirmed to be at $z=2$.

Given the significant use of photometric redshifts required to carry out this work, we are not able to distinguish galaxies at $z=$ 2 from galaxies at $z=2.07$, and we thus retain sources belonging to the $z=2.07$ structure in our sample of candidate members (unless a spectroscopic redshift is available). On the other hand, as discussed in detail in the Gobat et al. (2013) companion paper, the $z=2.07$ redshift peak seems to be associated with a large-scale, less prominent diffuse structure, which does not significantly contribute to the overdensity in the central cluster region studied here, and thus is not likely to significantly affect the results presented in this work.

Wide multiwavelength coverage and high-resolution restframe optical imaging allow us to study in detail the fundamental properties of cluster galaxies already 10 billion years ago. In particular, in this paper we focus on the identification of a population of passive candidate members, and on the investigation of their structural properties. The trademark cluster-core galaxies up to $z \sim 1$, massive galaxies with low star formation are in fact expected to be significantly rarer by $z \sim 2$. At this epoch, not only might they still be forming many of their stars, but also the velocity dispersion in cluster cores is still low enough that merging-driven mass assembly can play an important role. Although conclusive evidence is still lacking, the early merging of already gas-poor galaxies in an overdense environment might also affect the structural properties of the resulting massive passive systems, producing galaxies that are structurally more evolved than their field counterparts. While the statistics are still poor, Cl J1449+0856 offers one of the very rare chances to study passive earlytypes very close to their formation epoch, together with still actively forming galaxies, in an already relatively evolved cluster core.
Throughout this work, we assume $\Omega_{M}=0.3, \Omega_{\Lambda}=$ $0.7, \mathrm{H}_{0}=70 \mathrm{~km} \mathrm{~s}^{-1} \mathrm{Mpc}^{-1}$, and a Salpeter (1955) initial mass function (IMF). Magnitudes and colors are in the $\mathrm{AB}$ system.

\section{DATA AND SAMPLE SELECTION}

\subsection{Catalogs and Derived Quantities}

\subsubsection{Photometry}

We use photometry measured from imaging in the $U, V$ (VLT/FORS), $B, R, i, z$ (Subaru/Suprime-Cam), $Y, J, H, K_{s}$ (Subaru/MOIRCS, plus additional VLT/ISAAC data for $J$ and $\left.K_{s}\right)$, F140W (HST/WFC3), and 3.6, $4.5 \mu \mathrm{m}$ (Spitzer/IRAC) bands. Sources were extracted with SExtractor (Bertin \& Arnouts 1996) on the F140W image, and photometry was measured in two ways, producing two independent multiwavelength catalogs. One catalog is based on aperture photometry (using a 1".5 aperture) measured with SExtractor, corrected for the different resolution of the images by using aperture corrections estimated on each image from the growth curve of point-like sources. The other catalog is based on photometry measured on each image using GALFIT (Peng et al. 2002, 2010a), fitting point-spread function (PSF)-convolved Gaussian profiles at the position of the F140W-detected sources. While the two approaches yield broadly consistent measurements, in most cases aperture photometry will be more accurate. ${ }^{10}$ On the other hand, and especially in crowded fields typical of a cluster core, blending may be a severe issue and the second approach offers a way to deal with it. In order to take this into account, in the following we will use both catalogs as described in detail in Section 2.1.2.

In the following, we select a sample with $m_{140}<25.7$ (corresponding to the $10 \sigma$ limit in a $1^{\prime \prime}$ aperture), within an area of $\sim 3.3 \mathrm{arcmin}^{2}$ uniformly covered in the WFC3/F140W imaging. This catalog contains $\sim 370$ objects. Seven pointlike sources (in the F140W image) down to $m_{140} \sim 22$ were removed; at fainter magnitudes, we further removed $\sim 30$ sources that may be stars based on their $B z K$ colors (Daddi et al. 2004). The inclusion or removal of these sources has no impact on the results of this work. The galaxy sample we use in the following sections thus contains $\sim 330$ galaxies down to $m_{140}=25.7$.

\subsubsection{Photometric Redshifts}

From the 13-band photometry, we estimated photometric redshifts (photo- $z$ s) with EAZY, using the standard set of templates (Brammer et al. 2008, 2011; Whitaker et al. 2011). Before the actual photo- $z$ estimation, we determined possible offsets in the photometry in different bands by iteratively comparing the best-fit versus measured photometry at fixed (spectroscopic) redshift (e.g., Capak et al. 2007; Ilbert et al. 2009) for a sample of $\sim 110$ sources with redshifts measured from WFC3 grism spectroscopy over the whole WFC3-covered field ${ }^{11}$ (Gobat et al. 2013). Systematic offsets between measured photometry and the photometry from the best-fit model spectral energy distribution

\footnotetext{
10 Note that this is not a general statement: based on simulations, it is true for the specific approach and settings we use. A further indication of the generally higher accuracy of aperture photometry in our case comes from photo- $z$ performance as discussed in Section 2.1.2.

11 In this spectroscopic sample of 110 sources, 94 objects are located in the uniformly-covered WFC3 image area used in the following analysis of galaxy populations; the remaining objects are located in the external part of the WFC3 image.
} 
(SED) for spectroscopic sources can be attributed to different causes including zero point and/or aperture correction errors, as well as model uncertainties. ${ }^{12}$ In the following, we use photometry corrected for these systematic offsets; we note that the offsets determined for the two independent photometric catalogs (SExtractor- and GALFIT-based) are generally consistent within $\sim 10 \%$.

The interquartile redshift range of the spectroscopic sample is $z=[1.1-2.0]$ (with redshifts up to $z \sim 3$ ), and its interquartile $m_{140}$ range is $m_{140}=$ [22.7-24.3] (reaching up to $m_{140} \sim$ 25.5). Therefore, while compared to the full $m_{140}<25.7$ sample spectroscopic sources are obviously typically brighter, the spectroscopic sample can still be considered generally representative of sources in the magnitude and redshift range studied here.

Photo- $z$ S were determined for both the SExtractor- and GALFIT-based catalogs. For the SExtractor catalog, they were also determined excluding the IRAC bands. The comparison of these three different photo- $z$ determinations was used to improve the photo- $z$ accuracy, by selecting for each source the most appropriate photo- $z$ estimate as detailed below. Comparing with the spectroscopic catalog, we find that photo- $z \mathrm{~s}$ determined from aperture photometry including IRAC bands show the least scatter, but also a significant fraction of outliers (almost 10\%). For sources in the magnitude range typical of our spectroscopic sample, outliers may be due to fatal errors and/or degeneracies (e.g., a double-peaked photo- $z$ probability distribution). Such conditions may be produced or worsened by systematic offsets in the photometry due to bad resolution (and thus contamination by neighboring sources). In such cases, the GALFIT-based photometry-and thus the derived photo- $z$ - may be more accurate than SExtractor aperture photometry, as discussed above.

We identified sources potentially affected by neighbor contamination by selecting objects in the $K_{s}$ and $3.6 \mu \mathrm{m}$ bands that fell in the circle containing $99 \%$ of the flux of a different source. ${ }^{13}$ In the area and magnitude range that we use in this work, about $10 \%$ of our sample is classified as potentially contaminated at $K$-band resolution $\left(\sim 0^{\prime \prime} .65\right)$, and $\sim 50 \%$ at the IRAC resolution $\left(\sim 2^{\prime \prime}\right)$.

For uncontaminated sources, we use photo- $z$ s from the SExtractor catalog. We also use SExtractor-based photo- $z \mathrm{~s}$ when only the IRAC photometry is flagged as potentially contaminated and photo- $z$ 's with and without IRAC bands are consistent. For the remaining sources (about $20 \%$ of the sample) photo- $z$ s from the GALFIT catalog were used.

We test this approach on the spectroscopic sample, although the fraction of potentially contaminated sources in this sample is, as it may be expected, smaller than in the full sample $(30 \%$ vs. 50\%). In spite of this, we find that we can significantly reduce the fraction of catastrophic outliers while retaining the higher photo- $z$ accuracy obtained with aperture photometry for the majority of sources. The final photo- $z$ catalog we use has a scatter (as estimated with the normalized median absolute deviation (NMAD), Hoaglin et al. 1983) of 5.7\% in $\Delta z /(1+z)$ and $3 \%$ catastrophic outliers $(|\Delta z| /(1+z)>0.2$; at least half of these outliers have a "less reliable" spectroscopic redshift

\footnotetext{
12 For our catalogs and with our settings, in most cases offsets were $<10 \%-20 \%$, except for bands with large uncertainties on the photometric zero point (as often suggested also by comparing colors of stars with stellar templates) and/or on the instrument response function.

13 This approach is quite conservative in that it does not make assumptions about the relative flux of the neighbors and thus includes potential contamination from much fainter sources.
}

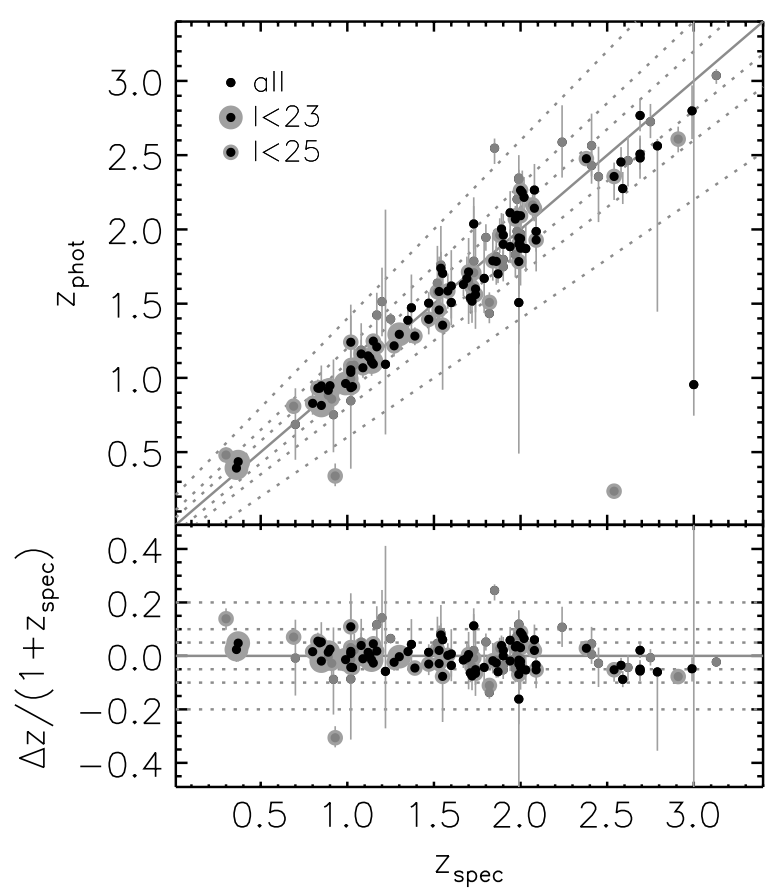

Figure 1. Comparison of photometric and spectroscopic redshifts for the available spectroscopic sample. All available spectroscopic redshifts are shown, but secure and less reliable $z_{\text {spec }}$ determinations are shown as black and gray dots, respectively. The lower panel shows $\left(z_{\text {phot }}-z_{\text {spec }}\right) /\left(1+z_{\text {spec }}\right)$ vs. $z_{\text {spec }}$. In all panels, the solid line traces $z_{\text {phot }}=z_{\text {spec }}$ and the dotted lines show a $\Delta z /(1+z)$ of $5 \%, 10 \%$, and $20 \%$. Thin and thick gray circles around symbols highlight sources brighter than $I<25$ and $23 \mathrm{mag}$, respectively, as indicated in the legend.

determination; see Figure 1). The comparison of photometric redshifts with the available spectroscopic sample is shown in Figure 1. For comparison with other studies, we note that, thanks to the WFC3 slitless spectroscopy, the spectroscopic sample we use is considerably deeper than those generally obtained from ground-based spectroscopy. The median I-band magnitude of our spectroscopic sample is $\sim 25$, with almost $80 \%$ of the sample fainter than $I=24$. For instance, considering only spectroscopic sources brighter than $I=25(23)$, the NMAD scatter of $\Delta z /(1+z)$ would be $<4.5 \%(<2.5 \%)$.

\subsubsection{Stellar Masses}

Stellar masses were determined with FAST (Kriek et al. 2009) on the 13-band $U$ to $4.5 \mu \mathrm{m}$ photometry, using Bruzual \& Charlot (2003, hereafter BC03) delayed exponentially declining SFHs $\left(\psi(t) \propto\left(t / \tau^{2}\right) \exp (-t / \tau)\right)$ with $0.01<\tau<10 \mathrm{Gyr}$, solar metallicities, a Salpeter IMF, and the Calzetti et al. (2000) reddening law with $E(B-V)$ up to 1 mag. Stellar masses were independently derived for both the SExtractor and the GALFIT catalogs: for sources where contamination was expected to significantly affect the aperture-based SED, as discussed concerning photo- $z \mathrm{~s}$ in Section 2.1.2, stellar masses from the GALFIT catalog were used.

Masses from the SExtractor catalog were corrected to "total" masses using the ratio between AUTO and aperture flux in the detection image (F140W). For the $m_{140}<25.7$ sample, more than $90 \%$ of the objects have a correction lower than $50 \%$, and only $\sim 3 \%$ of sources have correction factors $\gtrsim 2$. While this approach corrects for the bulk of the flux loss, we note that it still relies on approximating the total flux with FLUX_AUTO, and it is based on just one band, and thus neglects any color 
gradient within the galaxy. In this respect, we note that the systematic offset between stellar masses from the GALFIT catalog and the two SExtractor catalogs (with and without IRAC photometry, corrected to total masses) is less than $0.1 \mathrm{dex}$, with a scatter of up to $\sim 0.3$ dex. Leaving the metallicity as a free parameter and using exponentially declining SFHs rather than delayed exponentials introduces no systematics for the overall sample and a further scatter of less than 0.1 dex in stellar mass (which is small compared to the scatter estimated above at fixed metallicity and SFH). With respect to the choice of the SFH, we note that it has been shown how other forms of SFHs might be more appropriate for star-forming galaxies at high redshift (Maraston et al. 2010; Papovich et al. 2011). For what directly concerns this work, rising or constant (possibly truncated) SFHs would change the stellar masses of our targets negligibly, and in any case well within the estimated errors. Other parameters which may be more affected by the SFH choice (such as, notably, star formation rates and the ages of star-forming galaxies) are not used in this paper.

On the other hand, using Maraston (2005, hereafter M05) rather than $\mathrm{BC} 03$ models would produce stellar masses systematically smaller (overall for the sample of interest) by a factor $\sim 0.15$ dex, with a scatter of $\sim 0.15 \mathrm{dex}$; this is discussed in detail below, where relevant. After accounting for the overall $0.15 \mathrm{dex}$ systematic offset, the stellar mass determinations with $\mathrm{BC} 03$ and M05 models (with metallicity either fixed to $Z_{\odot}$ or allowed to vary within a factor of two from $Z_{\odot}$ ) are consistent within a factor of at most two for $>90 \%$ of the sample of interest.

Finally, the median formal error on stellar masses for our sample of candidate members (see Section 2.3) is $0.2-0.3 \mathrm{dex}$ (or $0.1-0.15$ dex for $m_{140}<24.5$ ). In summary, we thus estimate a typical accuracy of about a factor of two for the stellar mass determination for our targets.

\subsection{Morphological Analysis}

A rough indication of galaxy structure (early type versus late type), effective radius, and ellipticity was obtained by modeling the two-dimensional surface brightness distribution. We used GALFIT (version 3), assuming a single Sersic profile for each F140W-detected source. The modeling was performed on the WFC3 F140W image, which has the best resolution within our data set and probes rest-frame optical light (approximately the $B$ band) at the cluster redshift. We used a PSF built from the data using median stacking of six high $\mathrm{S} / \mathrm{N}$ stars in the field. The background was measured and subtracted locally over the whole image, and was fixed to zero in the fit. The whole image was fit multiple times, split in overlapping cutouts, ${ }^{14}$ modeling simultaneously all sources in the cutout.

In order to estimate the reliability of the results as a function of magnitude and profile type for the image and fitting settings that we used, we carried out simulations of the fitting procedure by adding synthetic sources in blank parts of the image. Sources with a range of magnitudes, $n_{\text {Sersic }}$, radius, ellipticity, and position angle were added and then fit with GALFIT using the same procedure used for real objects. These simulations provide an estimate of the reliability of our analysis in somewhat "optimistic conditions," since they assume that sources are relatively isolated, regular Sersic profiles, convolved with the

\footnotetext{
14 For each source, the final estimate of each parameter was calculated as the median among all fits with residuals of $<25 \%$ on at least $90 \%$ of the $\mathrm{S} / \mathrm{N}>10$ pixels. Overall, $3 / 4(1 / 2)$ of the sources in the magnitude range of interest $\left(m_{140} \lesssim 24.5\right.$; see below) have results derived from the median of at least 5 ( $>10)$ different fits, respectively.
}

same PSF that we use for the actual fitting. The input flux is recovered within $10 \%$ down to $m_{140}=24.5$ (corresponding to $\sim 30$ times the noise in a $1^{\prime \prime}$ aperture). At this magnitude, these simulations suggest that the error on the semi-major axis is $\sim 10 \%, 15 \%$, and $25 \%$ for profiles with $n_{\text {Sersic }}<1.5,1.5-3$, and $>3$, respectively, while the error on the Sersic index is between $\sim 25 \%$ for late-type profiles and $\sim 30 \%$ for early-type profiles. In addition, as it is known from previous work (e.g., Trujillo et al. 2006b; Sargent et al. 2007; Pannella et al. 2009), at faint magnitudes the Sersic index of high-Sersic profiles tends to be underestimated. With our settings, at $m_{140}=24.5$, our simulations suggest a median offset in the Sersic index of about $-10 \%$ for early-type profiles (disk-like profiles are unaffected). This systematic underestimation is negligible down to $m_{140} \sim 24$, where errors on semi-major axis are $<5 \%, 20 \%$, and errors on $n_{\text {Sersic }}$ are $<15 \%, 20 \%$, for low- and high-Sersic profiles, respectively. All parameters, for all kinds of profiles, are recovered at better than $10 \%$ down to $m_{140} \sim 23$ (corresponding to $\mathrm{S} / \mathrm{N} \sim 100$ in a $1^{\prime \prime}$ aperture).

Based on these simulations, we set $m_{140}=24.5$ as the limiting magnitude where we consider our surface brightness modeling reliable. Beside the general $m_{140}<25.7$ sample, we will thus consider this $m_{140}<24.5$ sample for the morphological analysis. Galaxy sizes quoted in the following are the circularized half-light radii, calculated from the GALFIT-based parameters as the effective semi-major axis times the square root of the axis ratio.

\subsection{The Candidate Member Sample}

A spectroscopic redshift is measured for about one-fourth of the $m_{140}<25.7$ sample, and for $\sim 45 \%$ of the $m_{140}<24.5$ sample. Based on the available spectroscopy, and otherwise on the photo- $z$ analysis, we thus determine which sources in our sample are (candidate) cluster members.

We select as spectroscopic members all sources with a spectroscopic redshift $1.97<z<2$.01. All other spectroscopic sources are considered interlopers. From the spectroscopic sample of Gobat et al. (2013), we retain 14 cluster members in the area studied in this work. One source in the $1.97<z<2.01$ range, close to the edge of the studied area, is not in our catalog as it is close to a bright star. From its spectrum, it is classified as a star-forming source and given its position more than $600 \mathrm{kpc}$ away from the cluster center, it would not alter (if anything, it would reinforce) the conclusions of this study. Furthermore, an X-ray-detected AGN classified as a cluster member in Gobat et al. (2013) is not included in our member sample. This source appears to have a very close neighbor $\sim 0$ '.5 away (with undetermined redshift), producing a likely significant contamination to its observed SED. Assuming that both sources belong to the cluster, and that the measured photometry is not significantly affected by the emission of the AGN, the total observed SED produced by both sources would suggest a dusty star-forming stellar population. The morphology of the AGN host appears very compact and essentially unresolved (although its magnitude is close to the limit where we can carry out a reliable morphological analysis). Because of the considerable uncertainties in determining the properties of this source, we will not consider it in the following analysis of galaxies in $\mathrm{Cl}$ J1449+0856.

For all sources without an available spectroscopic redshift, we rely on photo-zs. For determining membership by photo-zs, we decided not to purely rely on the redshift probability distribution estimated by EAZY for each object. We adopted instead a hybrid 
approach, taking into account also the "empirical" photo- $z$ uncertainty as independently determined by comparison with the spectroscopic sample. By analyzing photo- $z$ results for spectroscopic sources (for the full spectroscopic sample, not just cluster members) as a function of the photo- $z$ probability distribution and the quality of the best fit, we first estimated the likely reliability of a photo- $z$ given its odds and $\chi^{2}$ calculated by EAZY (within our catalog). Based on such a comparison, we deemed as "most reliable" photo- $z$ s with odds $>98 \%$ and a $\chi^{2}$ within three times the median $\chi^{2}$ in our catalog. To the other extreme, we defined as "unreliable" photo- $z$ s with odds lower than $95 \%$, or with a $\chi^{2}$ value worse than six times the median $\chi^{2}$.

For the purpose of membership assignment, the full sample was then split into three classes of objects, identified as interlopers, "likely" candidate members, and "possible" candidate members (which are lower-priority candidates, mainly due to a more uncertain photo- $z$ determination). Based on the observed scatter of $\Delta z /(1+z)$ (Section 2.1.2), and considering the estimated low fraction of catastrophic failures, all galaxies with a photo- $z$ beyond $2 \sigma$ from the cluster redshift are considered interlopers, except those within $3.5 \sigma$ and with a photo- $z$ deemed unreliable, which are retained as possible members. All galaxies with a photo- $z$ within $2 \sigma$ of the cluster redshift are considered possible members, unless they have a highly reliable photo- $z$ and an integrated photo- $z$ probability distribution within $1 \sigma$ of the cluster redshift ${ }^{15} P\left(z_{\mathrm{cl}}\right)>30 \%$, which classifies them as likely members. All galaxies with a photo- $z$ within $1 \sigma$ of the cluster redshift are selected as likely members.

Likely members make up $\sim 15 \%$ of the full sample, and an additional $15 \%$ of objects are possible members. The remaining $\sim 70 \%$ of the full sample is rejected as foreground $(\sim 55 \%)$ or background $(\sim 15 \%)$ interlopers.

For comparison with other studies, we note that $\sim 3 / 4$ of galaxies selected as likely members have $P\left(z_{\mathrm{cl}}\right)>30 \%$ (with $P\left(z_{\mathrm{cl}}\right)>20 \%$ for almost all likely members), and in turn $>80 \%$ of galaxies with $P\left(z_{\mathrm{cl}}\right)>30 \%$ are classified as likely members, making the likely-member selection roughly similar to that used by, e.g., Tran et al. (2010) and Papovich et al. (2012). On the other hand, possible members have $P\left(z_{\mathrm{cl}}\right)$ as low as $\sim 1 \%$, with an interquartile range of $P\left(z_{\mathrm{cl}}\right)$ of about $6 \%-22 \%$. The inclusion of possible members thus brings our selection closer to more conservative $P\left(z_{\mathrm{cl}}\right)$-based criteria adopted by other studies (e.g., Tanaka et al. 2013; Raichoor \& Andreon 2012). In fact, in this work, galaxies with a $P\left(z_{\mathrm{cl}}\right)$ larger than $10 \%$ are essentially always included in the candidate sample (as either likely or possible members), unless they are spectroscopic interlopers. Overall, the selection we use is thus quite conservative, which is reflected in the considerations about completeness and contamination discussed below.

\subsubsection{Completeness and Contamination of the Candidate Member Sample}

Figure 2 shows the redshift distribution in the considered area, down to $m_{140}=24.5$ and 25.7 , and highlights the different contributions of interlopers versus possible and likely candidate cluster members. The presence of the cluster on the underlying field redshift distribution is clearly visible, even if this distribution is largely based on photo-zs. Comparing with measurements in wide fields (e.g., Muzzin et al. 2013), the

15 That is, $P\left(z_{\mathrm{cl}}\right)=\int_{2-3 \times 0.057}^{2+3 \times 0.057} p(z) d z$.

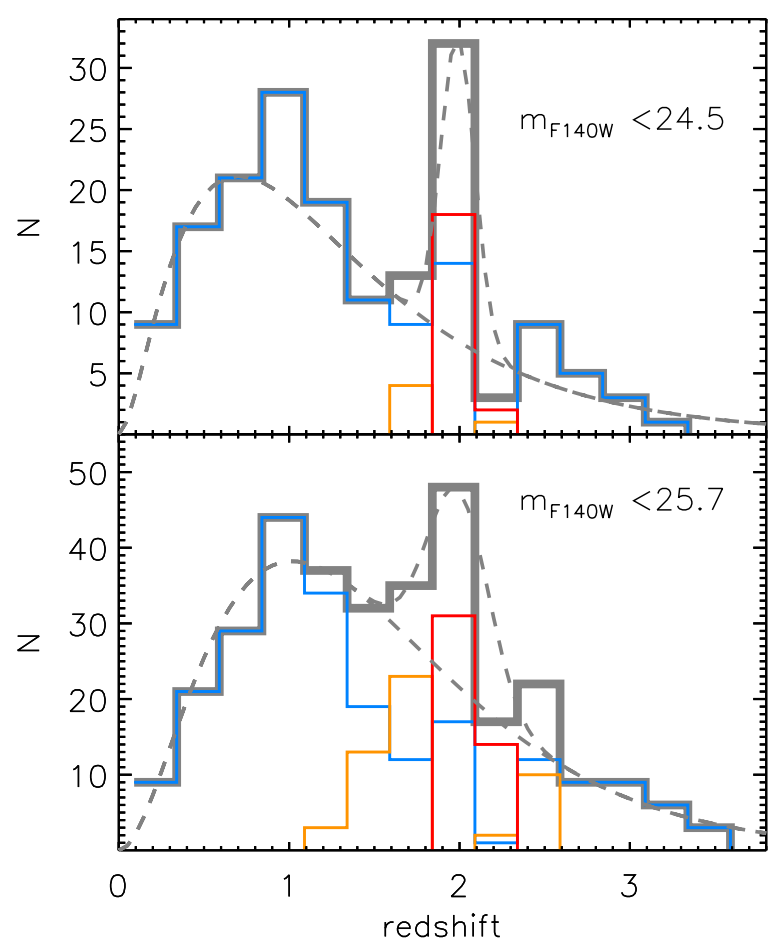

Figure 2. Redshift distribution in the cluster field. Gray lines show the distribution (of photometric redshifts, or spectroscopic redshifts where available) of all sources in the target area down to $m_{140}=24.5$ and 25.7 (upper and lower panels, respectively). The contributions of galaxies identified as interlopers, "possible," and "likely" cluster members, as described in the text, are shown as blue, orange, and red lines, respectively. Dashed gray lines in both panels show a modeling of the redshift distribution in this field, with and without the cluster contribution (see the text).

(A color version of this figure is available in the online journal.)

comoving number density of massive $\left(>10^{10} M_{\odot}\right)$ galaxies at $1.7<z<2.1$ (the photo- $z$ range most affected by cluster members) in the area within $150 \mathrm{kpc}$ from the cluster center is about 30 times larger $\left(\Delta \log \left(n / \mathrm{Mpc}^{3}\right)=1.49 \pm 0.15 \mathrm{dex}\right) .{ }^{16}$

We note that, by comparison with the spectroscopic sample, our selection of candidate cluster members is highly complete (all 19 spectroscopically confirmed cluster members in the area probed by the WFC3 grism data would be classified as likely candidate members based on their photo- $z$ s). On the other hand, as a tradeoff with completeness, the sample of candidate members is significantly contaminated by interlopers.

As a rough estimate of such contamination, we find that by comparison with the spectroscopic sample, about half of the sample of "likely" cluster members would be interlopers. We note that this fraction increases significantly, possibly to $\sim 80 \%$, for "possible" members, for the obvious reason that, by selection, this sample is made up of objects with a photo- $z$ more distant from the cluster redshift, and often poorly constrained. For such (typically faint) sources, not only it is difficult to obtain a reliable redshift estimate, but it is also hard to estimate photo- $z$ accuracy and contamination. On the other hand, as we show below, the vast majority of these uncertain candidates are

\footnotetext{
16 We verified that the comoving number density measured in our field in the $1<z<1.5$ range (i.e., not affected by the cluster), is in excellent agreement with that measured by Muzzin et al. (2013). Even in the $1.7<z<2.1$ range affected by the cluster, the comoving number density of massive galaxies beyond $200 \mathrm{kpc}$ from the cluster center is only a factor $\sim 4$ times the density of the general field $\left(\Delta \log \left(n / \mathrm{Mpc}^{3}\right)=0.58 \pm 0.15 \mathrm{dex}\right)$.
} 
low-mass star-forming galaxies that do not enter our masscomplete samples, and have generally little weight in our conclusions. We finally note that spectroscopic interlopers classified as likely and possible members are generally close to the cluster redshift ( $\gtrsim 90 \%$ at $1.8<z<2.1$ and $1.4<z<2.7$, for likely and possible members, respectively).

As a further check of the relevance of contamination, we model the redshift distribution in Figure 2 with an $f(z)=$ $C \times\left(\beta z^{2} / \Gamma(3 / \beta) z_{0}^{3}\right) e^{-\left(z / z_{0}\right)^{\beta}}$ (Brainerd et al. 1996) for the field plus a Gaussian centered at $z=2$ for the cluster (dashed gray lines in the figure). The modeling is only done for the purpose of estimating the cluster and field contributions in the $1.5<z<2.5$ redshift range, ${ }^{17}$ as relevant to membership determination.

For the $m_{140}<25.7$ sample (lower panel of Figure 2), the width of the Gaussian is fixed based on the estimated photo- $z$ scatter. From the modeling, we estimate that in the $1.5<z<2.5$ range about one-third of this sample should be made of cluster galaxies. Therefore, in this redshift and magnitude range, statistically we should have in our catalog about 40 cluster members. We have 14 spectroscopic members and 31 likely and 40 possible candidates. Assuming an estimated $50 \%$ contamination for likely members and $80 \%$ contamination for possible members, we expect $\sim 24$ actual members among the candidates, in close agreement with the statistical estimate.

For the $m_{140}<24.5$ sample, the photo- $z$ scatter is smaller $(\sim 5 \%)$, and many cluster members are spectroscopically confirmed. This results in a tighter Gaussian in the upper panel of Figure 2: we use $0.1<\sigma<0.15$. For this bright sample, we estimate that about $50 \%$ (and at least $40 \%$ ) of the galaxies should be cluster members. Thus, statistically, there should be $\sim 29$ (and at least 23) cluster members in this magnitude and redshift range in our catalog. Since we have 13 spectroscopic members and 12 candidates, this could suggest that, for the bright sample, our membership determination is less affected by contamination (as could be expected). Overall, this check confirms that our estimate of contamination for the whole sample is realistic; if anything, estimates might be somewhat too high for bright sources.

\subsubsection{Final Samples}

In the end, we have a sample of 96 (candidate) members down to $m_{140}=25.7: 14$ spectroscopic members, 31 "likely," and 51 "possible" candidates. ${ }^{18}$ Based on the considerations discussed above, we expect the whole sample of candidate members to include about 50 interlopers, for the most part (75\%) selected as possible members.

We stress that, because of the significant contamination of our candidate member sample by field galaxies at similar redshifts, in most of this paper we will not be able to investigate the detailed comparison of galaxy properties in cluster and field environment at $z \sim 2$. On the other hand, in spite of the significant contamination or in some cases thanks to the extensive WFC3 spectroscopy, some properties of the cluster

\footnotetext{
17 We use $\beta=0.72, z_{0}=0.17$ for $m_{140}<24.5$, and $\beta=1, z_{0}=0.51$ for $m_{140}<25.7$, but note that given the very small area we probe, and the contamination from the cluster itself, these data are not ideal for modeling $f(z)$. Thus, the parameters determined here should not be considered for general purposes.

18 Four more sources classified as possible members are embedded in the halos of bright objects and were excluded because of their unclear nature and severely corrupted photometry.
}

galaxy populations are clearly visible, even after dilution of their signal with field galaxies, as discussed below.

As discussed in Section 2.2, for all analyses involving the characterization of galaxy morphological structure, we limit our sample to objects brighter than $m_{140}=24.5$. The sample is thus reduced to $\sim 170$ sources, including 13 spectroscopic and 12 candidate members.

The mass completeness limits corresponding to the magnitudes $m_{140}=25.7$ and 24.5, estimated for an simple stellar population (SSP) formed at $z_{f}=5$, with solar metallicity and no dust reddening, are $\sim 8.5 \times 10^{9} M_{\odot}$ and $2.5 \times 10^{10} M_{\odot}$, respectively (Salpeter IMF).

\subsection{Passive and Star-forming Galaxies}

In order to broadly characterize the stellar population properties of individual sources, we split the sample into two classes of galaxies that are either essentially passively evolving or still actively forming stars. We initially assign galaxies to one class or the other based on their rest-frame $U-V$ and $V-J$ colors (e.g., Wuyts et al. 2007, hereafter UVJ classification), as calculated based on each galaxy's redshift and its observed SED (as selected in Section 2.1.2; spectroscopic redshifts are used where available). We use here the division between passive and active galaxies in the $U V J$ plane as adopted in Williams et al. (2009).

We then re-fit the observed SEDs of candidate members, at fixed redshift (photo- $z$ or spectroscopic value), using combinations of templates from two different libraries. The first library includes only $\mathrm{BC} 03$ passive templates (age $/ \tau>4.5$ and age $\geqslant$ $0.6 \mathrm{Gyr}$ ), with different metallicities, no dust attenuation, and a range of ages appropriate for the redshift range of the candidate members. This library is thus only appropriate for passive galaxies, in the relevant redshift range, with little dust attenuation. The second library includes only $\mathrm{BC} 03$ templates with constant SFHs, attenuated by dust with $E(B-V)$ up to 1.2, and with a range of ages appropriate for the redshift considered. This library may thus only describe actively star-forming populations with a broad range in dust attenuation, including highly reddened sources.

If we compare the $\chi^{2}$ of the best fits for cluster members obtained with these two libraries and with the EAZY standard templates, we generally find that more than half of the $U V J$ star-forming galaxies are best fit with the EAZY library, $40 \%$ are best fit with the constant SFH library, and only $4 \%$ have the lowest $\chi^{2}$ with the passive library. On the other hand, candidate members classified as $U V J$-passive are essentially never best fit by constant SFH templates (with the exception of a source close to the dividing line), and in $90 \%$ of cases have $\chi_{\text {PASSIVE }}^{2} \lesssim \chi_{\text {EAZY }}^{2}$ (we note that the best-fit EAZY SED can also be essentially passive, with the greatest contribution coming from templates of evolved populations). ${ }^{19}$ In the great majority of cases, this alternative classification based on the comparison of the SED fit with different libraries agrees with the $U V J$ selection. In very few cases, the two classifications do not agree (see Figure 3 ). These objects are often close to the dividing line of the $U V J$ plot, where it is thus particularly useful to also have a different approach. Alternatively, some of these objects are faint sources whose

\footnotetext{
19 When a spectroscopic redshift is not available, as discussed above, we fix the redshift to the photometric value. This is a sensible choice given that a photometric redshift is incomparably better constrained with the general template library. Nonetheless, we note that for most $(70 \%)$ of the passive sources, constant SFH templates give a poorer fit even if redshift is left as a free parameter.
} 


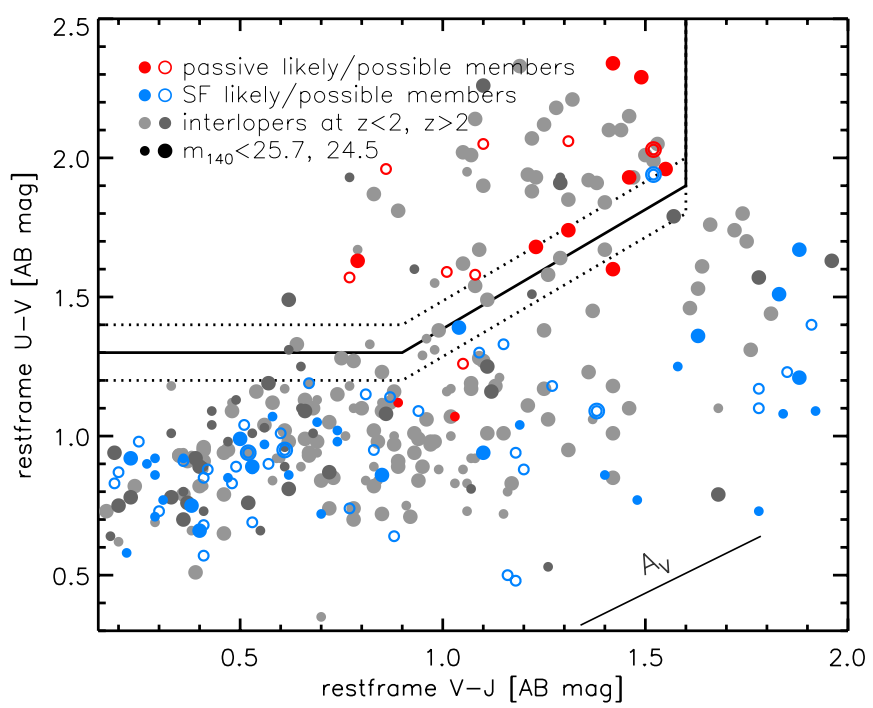

Figure 3. Rest-frame $U-V$ vs. $V-J$ color-color plot. Larger/smaller symbols show galaxies brighter than the two limits used $\left(m_{140}<24.5\right.$ and 25.7, respectively). The solid line shows the separation criterion between passive and star-forming galaxies at $z \sim 2$ as adopted in Williams et al. (2009)—dotted lines are, as a reference, $\pm 0.1 \mathrm{mag}$ around this limit. Interlopers in the foreground and background are shown as light and dark gray symbols. Colored symbols show cluster candidate members ("likely" and "possible" as full and empty circles), red for passive and blue for star-forming sources according to the SED classification-see the text for details.

(A color version of this figure is available in the online journal.)

SED is poorly constrained due to large photometric errors. In these few cases, we adopt the SED-based classification.

Our sample of candidate members is thus ultimately divided into 18 passive (6 secure members plus 4 likely and 8 possible candidates) and 78 actively star-forming galaxies (8 secure members plus 27 likely and 43 possible candidates).

\section{GALAXIES ASSOCIATED WITH THE CL 1449+0856 STRUCTURE}

As discussed above, our selection of candidate members should be highly complete but also significantly affected by contamination from interlopers. While it is impossible to remove this contamination based on photometric redshifts, we can at least statistically investigate some properties of the cluster galaxy populations that are strong enough not to be diluted by the significant presence of interlopers.

\subsection{Projected Distribution of Candidate Members}

We show in Figures 4 and 5 the projected distribution of candidate cluster members in the field. Note that both figures show all candidate members-according to the specific selection as labeled in individual panels_-and are thus affected by interloper contamination (as discussed in Sections 2.3.1 and 2.3.2). While the more uncertain "possible" members make up about half of the full $m_{140}<25.7$ candidate sample, their contribution is higher at lower masses, and goes down to $<30 \%$ and $25 \%$ for the $\log M / M_{\odot}>9.9$ and $\log M / M_{\odot}>10.4$ mass-complete samples highlighted in the figures. Accounting for spectroscopic members and the estimated contamination for "likely" and "possible" candidates, we estimate that these masscomplete samples are affected by an overall contamination of $<40 \%$ and $\sim 30 \%$, for $\log M / M_{\odot}>9.9$ and $\log M / M_{\odot}>10.4$, respectively.

Figure 4 shows individually all galaxies in the field, highlighting "possible" and "likely" candidate members, as well as the nature of their stellar populations as estimated from their SEDs (Section 2.4).

Figure 5 shows local density maps of the same sample of candidate cluster members $\left(m_{140}<25.7\right)$, as well as of the masscomplete sample $\left(\log M / M_{\odot}>9.9\right)$. These maps show more clearly the projected distribution of (candidate) cluster galaxies, the density enhancement around the cluster center (taken as
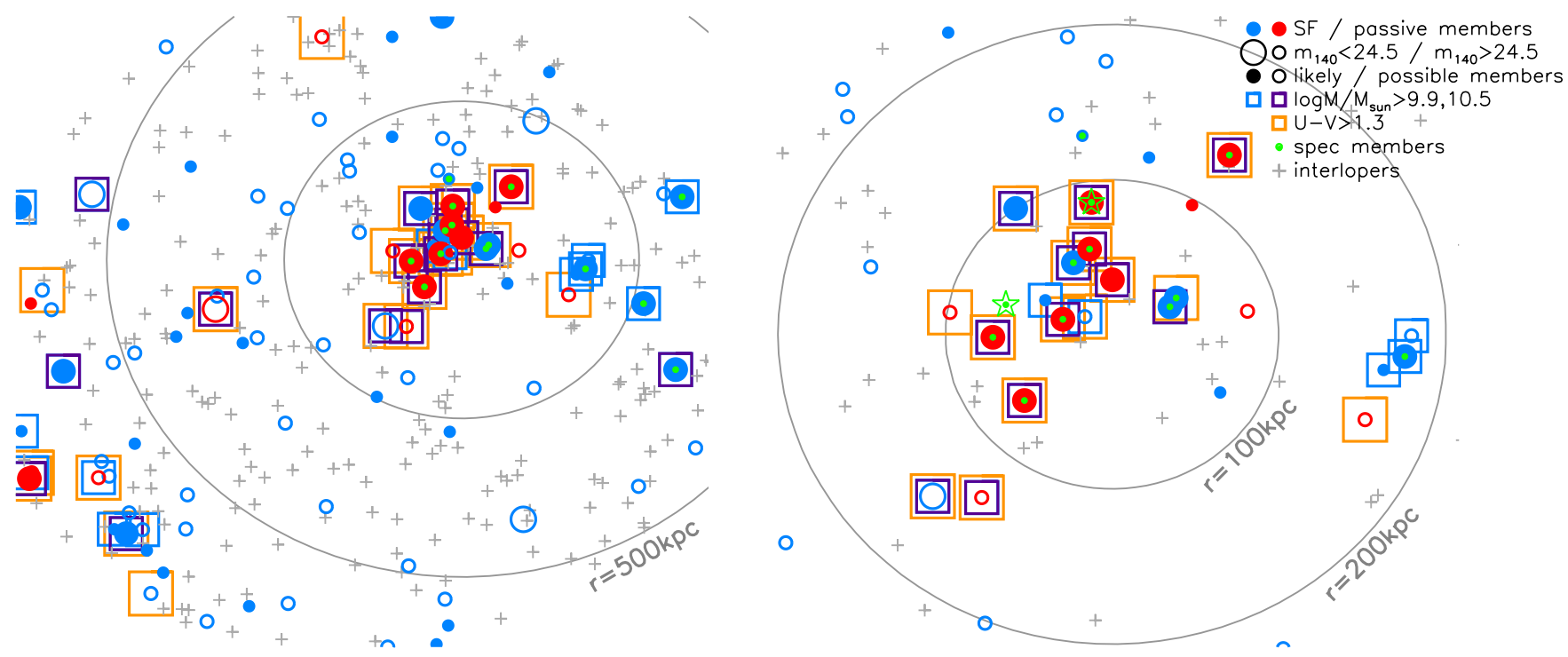

Figure 4. Left: the distribution of galaxies brighter than $m_{140}=25.7$ in the studied field. Interlopers are plotted as gray crosses, while passive and star-forming candidate members are highlighted in red and blue, respectively. Filled and empty circles show likely and possible candidates, and spectroscopically-confirmed members are marked with a small green point in the center. Large and small circles show sources brighter than $m_{140}=24.5$ and $m_{140}=25.7$, respectively. Yellow squares mark candidate members with rest-frame $U-V>1.3$, while blue and purple squares show the mass-complete samples of members more massive than $\log M / M_{\odot}=9.9$ and 10.4, respectively. Solid gray circles show clustercentric radii of 250 and $500 \mathrm{kpc}$ (proper) at the cluster redshift. North is up, east to the left. Right: a close-up of the left-hand panel in the cluster center. Symbols are the same, gray circles mark clustercentric radii of 100 and $200 \mathrm{kpc}$ (proper) at the cluster redshift. Two AGNs spectroscopically confirmed to belong to the cluster (Gobat et al. 2013) are marked by green stars.

(A color version of this figure is available in the online journal.) 

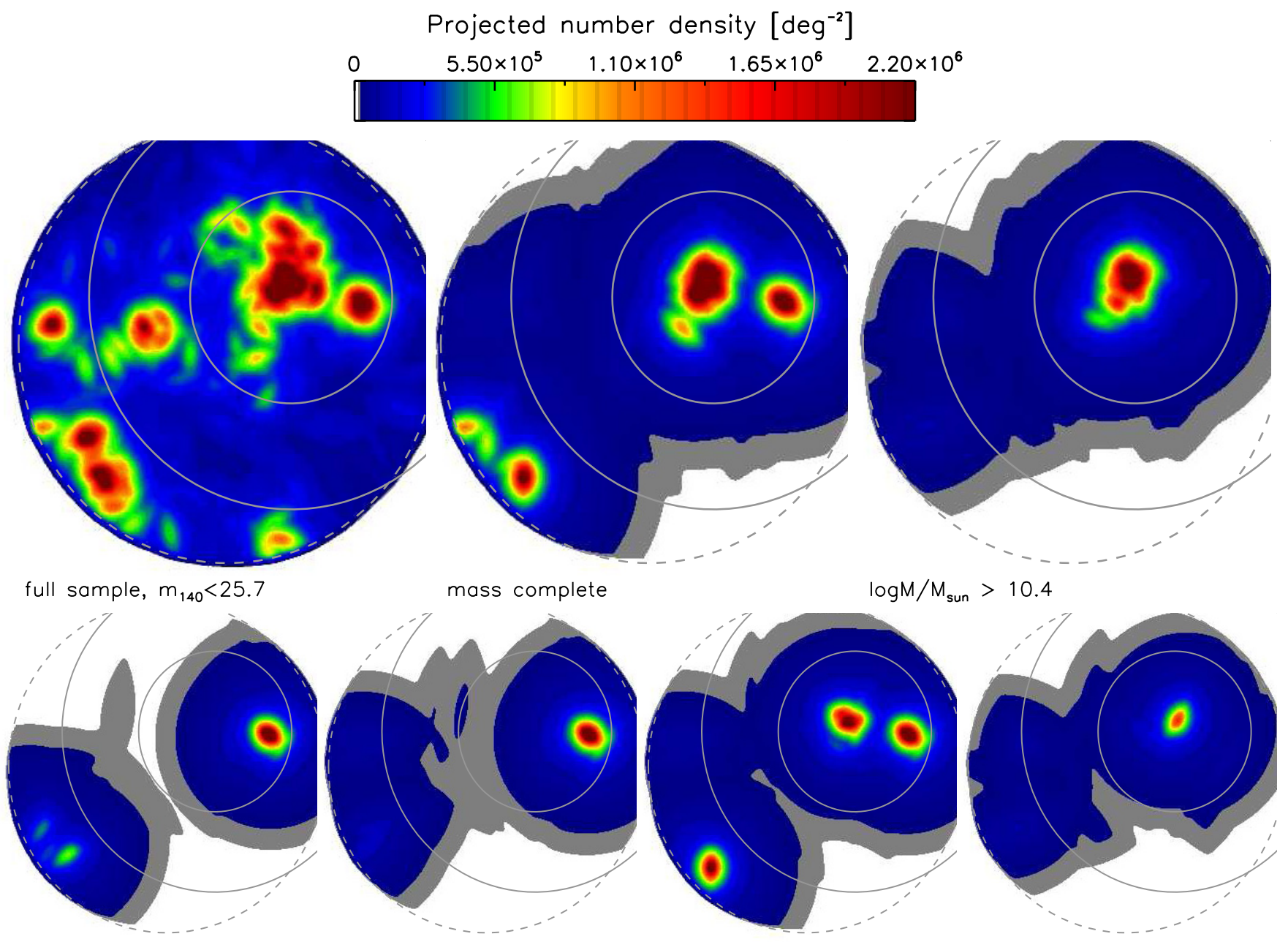

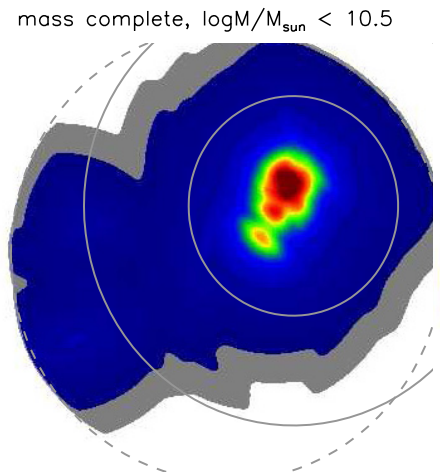

mass complete, $\log M / M_{\text {sun }}>10.5$

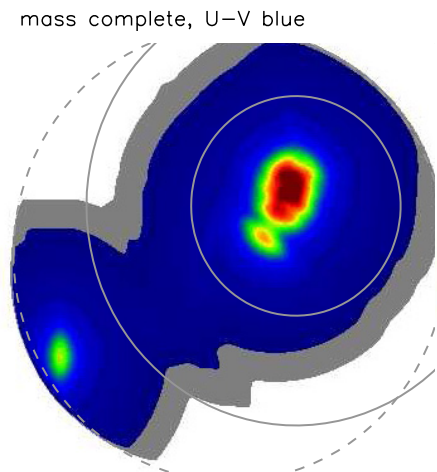

mass complete, $U-V$ red

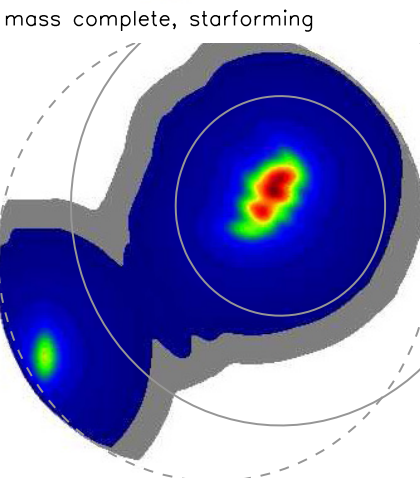

mass complete, quiescent

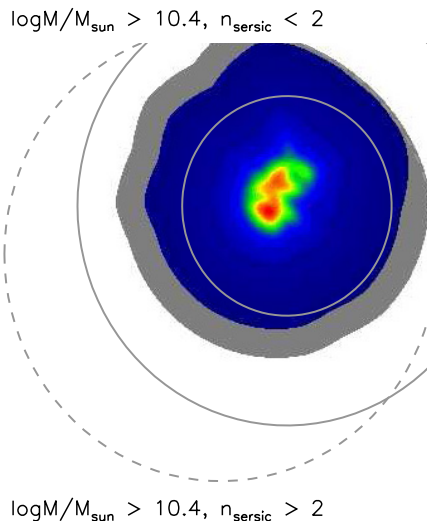

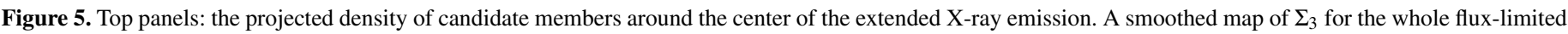

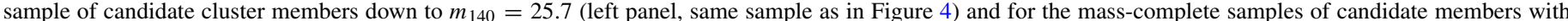

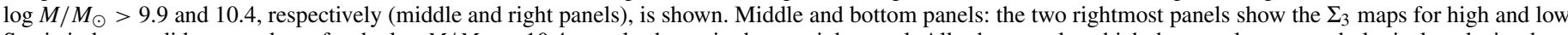

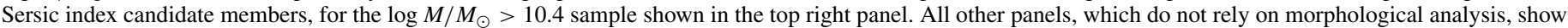

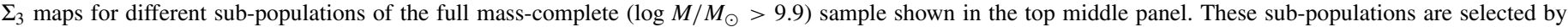

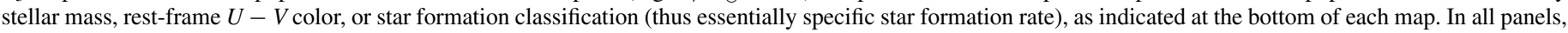

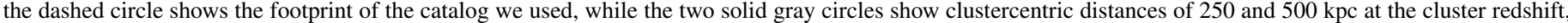

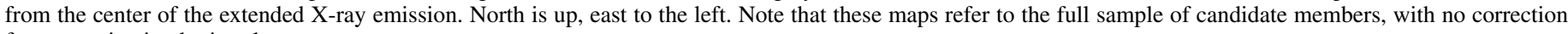
for contamination by interlopers.

(A color version of this figure is available in the online journal.)

the center of the extended X-ray emission, as quoted in Gobat et al. 2011), and possible surrounding structures. As an estimator of local (projected) density, we used the density based on the distance to the third nearest neighbor, $\Sigma_{3}$. We correct for edge effects by accounting for uncovered area within the distance to the third nearest neighbor, however, minor edge effects may still persist. Contours from the density map of the full flux-limited $m_{140}<25.7$ sample of candidate members (top left panel of Figure 5) are shown overlaid on the WFC3 F140W image in Figure 6. Figure 5 also shows local density maps of different sub-populations of the mass-complete sample to highlight similarities and differences in their projected distributions. 


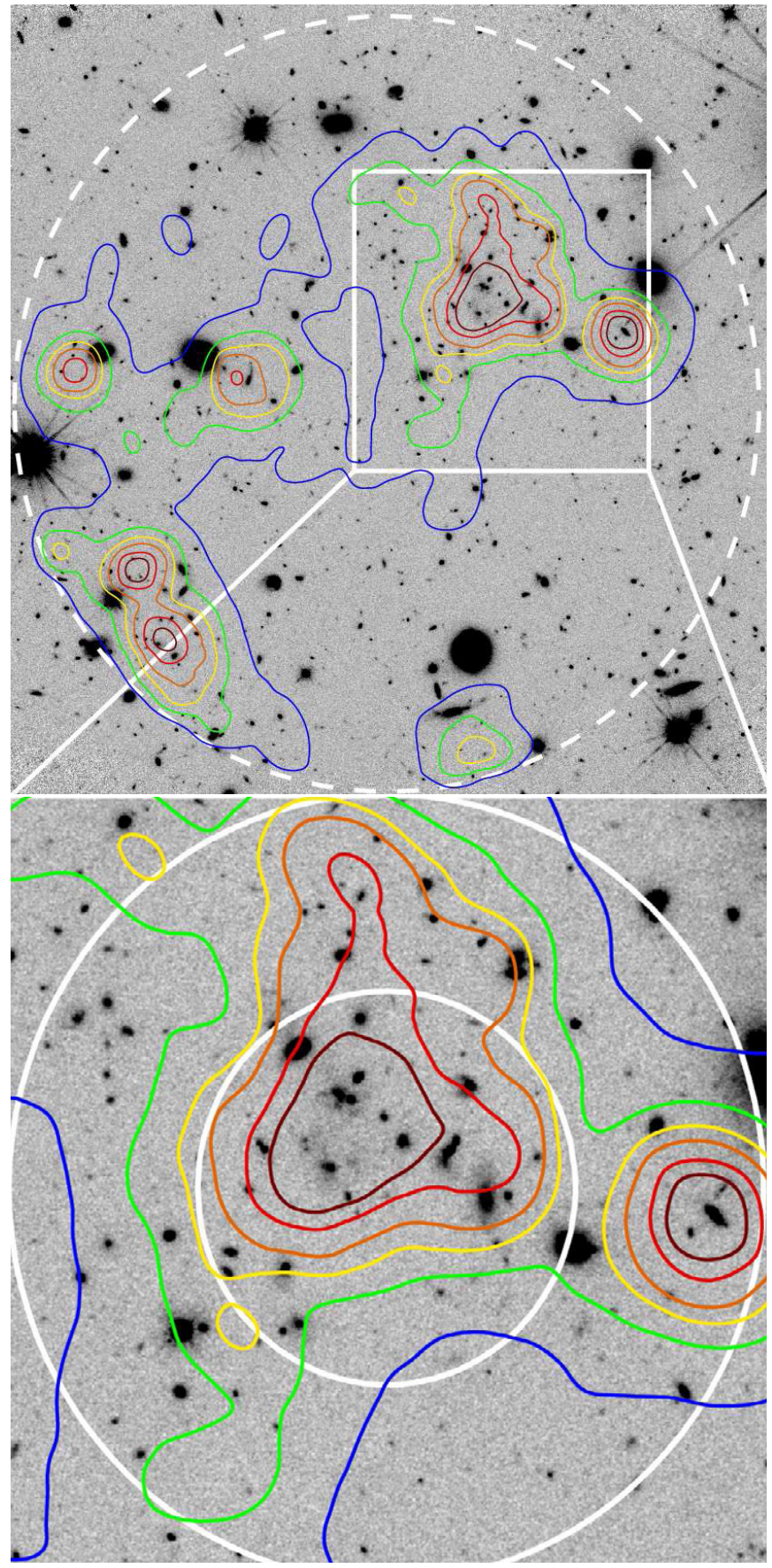

Figure 6. WFC3 F140W image of the studied area. Contours correspond to the density map of the full flux-limited sample of candidate members shown in Figure 5 (top left panel), with colors corresponding to projected number density levels in the same color scale. The dashed circle shows the footprint of the catalog we used, as in Figure 5. In the bottom panel, a close-up of the inner cluster region (white square in the upper panel) is shown. White circles show radii of 100 and $200 \mathrm{kpc}$ (proper) at the cluster redshift.

(A color version of this figure is available in the online journal.)

Figures 4 and 5 clearly show the characteristic nature of the galaxies in the central concentration, including many massive, red, passive sources within 100-150 kpc of the cluster center. West of the cluster center, these figures show an overdensity of galaxies of a seemingly different nature, less massive, starforming, and (where available) of late-type structure (Figure 7). Half of these galaxies are spectroscopically confirmed to be cluster members.
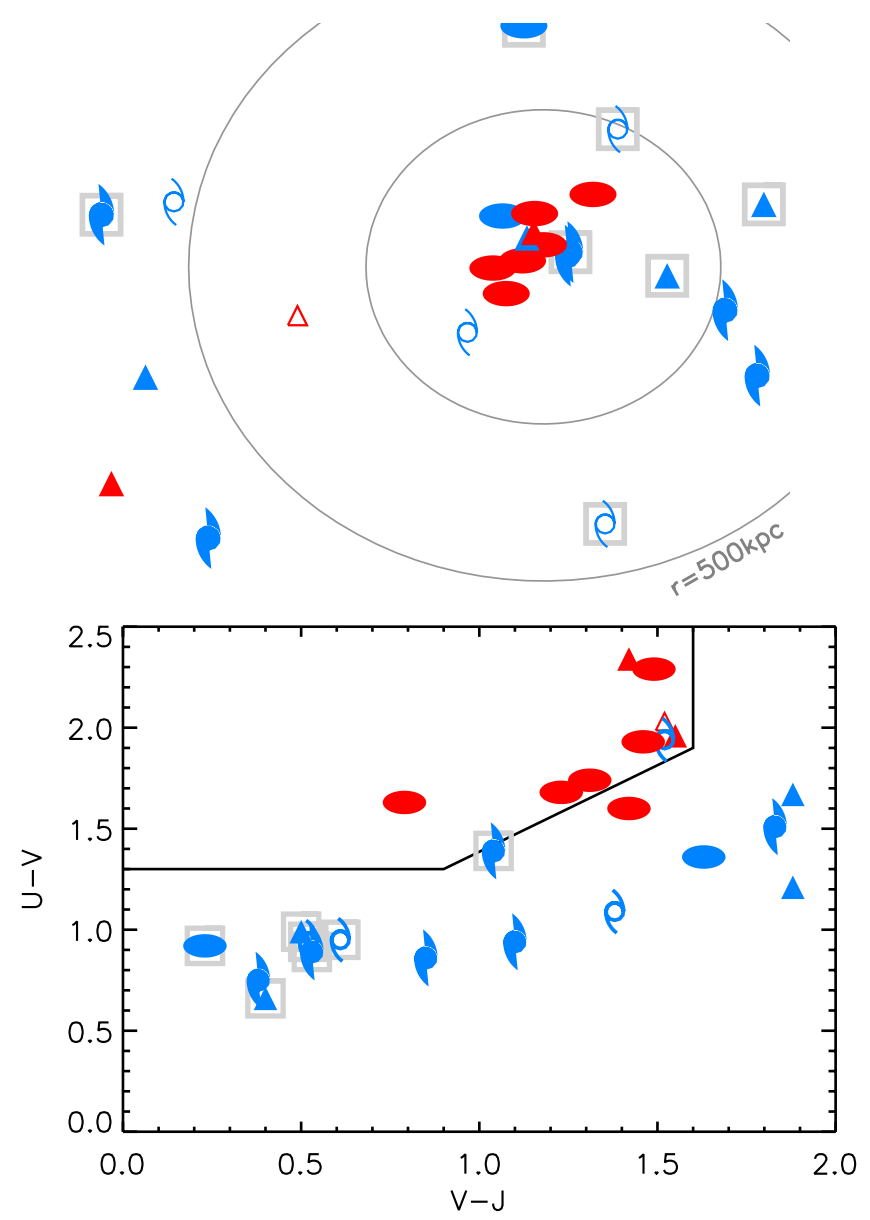

Figure 7. Projected distribution (top panel, as in Figure 4) and the $U V J$ restframe color-color plot (bottom panel) of the sample of candidate members brighter than the limit for morphological analysis $\left(m_{140}<24.5\right)$. This sample is flux limited, not mass complete: sources below the estimated mass completeness of $\log M / M_{\odot}=10.4$ are highlighted with gray squares. Solid/empty symbols show likely/possible members, respectively. Galaxies classified as passive or star forming are colored in red and blue, while galaxies with $n_{\text {Sersic }}$ higher or below 2 are shown as ellipses and spirals, respectively. Galaxies for which no acceptable fit could be obtained are plotted as triangles; visual inspection shows that only one, a passive source very close to the cluster center, might possibly be an early-type.

(A color version of this figure is available in the online journal.)

Another overdensity in the projected distribution of candidate members is located southeast of the cluster center. However, as Figure 4 shows, it is made in large part of candidates less likely to be at the cluster redshift, and it contains no spectroscopically confirmed members. The reality of this structure could not be confirmed with the current spectroscopic coverage, partly due to observational issues; it is located at the edge of the field fully covered by WFC3 grism spectroscopy with all four orientations, and is largely composed of faint sources.

Considering the mass-complete sample (blue and purple squares in Figure 4), and in spite of dilution due to interloper contamination, a concentration of massive, of optically red (rest-frame $U-V>1.3^{20}$ ), and of passive galaxies in the cluster core is evident. This central concentration appears to include mostly passive sources, but also some dust-reddened star-forming galaxies. These results seem to be largely stable

20 The rest-frame $U-V>1.3$ threshold, used here when referring to optically red sources, is close to the observed $Y-K$ color cut used in Gobat et al. (2011). 
against the inclusion of interlopers. In fact, due to the selection criteria, a large fraction of the less likely ("possible") members is made up of low-mass star-forming galaxies below the mass completeness limit (75\%, versus 50\% for "likely" members). More specifically, the concentration of massive, red, and passive galaxies in the cluster core does not depend on the inclusion of less likely members.

\subsection{Structural and Stellar Population Properties}

Passive systems, as well as more massive galaxies $\left(\log M / M_{\odot}>10.5\right)$, seem to be effectively segregated in the central cluster region, with two-thirds of these galaxies within $200 \mathrm{kpc}$ of the cluster center.

At least in the mass range probed by our morphological analysis $\left(\log M / M_{\odot}>10.4\right)$, this segregation is also evident for high-Sersic systems $(n>2)$, which are all within a clustercentric distance of $\sim 150 \mathrm{kpc}$, as shown in Figures 5 and 7.

This extends to a $z=2$ cluster previous results showing that, already before $z \sim 1$, the central regions of clusters and groups generally exhibit a segregation of more massive, older, or morphologically-evolved galaxies (e.g., among others, Rosati et al. 2009; Mei et al. 2012; Muzzin et al. 2012). In particular, the studies of Kurk et al. (2009), Tanaka et al. (2010b, 2013), and Papovich et al. (2010, 2012) of two $\mathrm{X}$-ray-detected low-mass clusters at $z \sim 1.6$ suggest that at least some overdense structures, even with relatively low masses and already at $z>1.5$, host in their core galaxy populations that are particularly evolved, in terms of their structure, stellar populations, and assembled stellar mass. Nevertheless, such evolved galaxies can be found to coexist in the cluster core together with a population of galaxies that are still actively forming (e.g., Tran et al. 2010). Indeed, we recall results from several studies suggesting that, even at $z \gtrsim 2$, some protocluster environments may already host galaxies more massive, with older stars and more evolved structure than their surroundings (e.g., Steidel et al. 2005; Kodama et al. 2007; Tanaka et al. 2010a; Hatch et al. 2011; Zirm et al. 2012; Spitler et al. 2012).

With respect to the comparison of structural and stellar population properties, we note a clear correlation in our sample of candidate members (at least in the probed mass range) between a high-Sersic profile and evolved host stellar populations (Figure 7) consistent with previous observations at similar redshifts in both field and high-density environments (e.g., Cimatti et al. 2008; Kurk et al. 2009; Wuyts et al. 2011; Cameron et al. 2011; Bell et al. 2012; Papovich et al. 2012; Tanaka et al. 2012; Patel et al. 2012). As discussed in more detail below, $70_{-20}^{+10} \%{ }^{21}$ of candidate passive members more massive than the $\log M / M_{\odot}>10.4$ threshold for morphological analysis have $n_{\text {Sersic }}>2$. A similar early-type fraction is found in the passive population of our sample of interlopers at $1.5<z<2.5$. Conversely, only $10_{-4}^{+20} \%$ of star-forming candidate members more massive than the same limit are classified morphologically as early type. For comparison, Papovich et al. (2012) find that about $80 \%$ of candidate members in the cluster XMM-LSS J02182-05102 at $z \sim 1.6$ have $n_{\text {Sersic }}>2$ (in a mass range similar to ours).

In turn, $\sim 75_{-20}^{+9} \%$ of the $\log M / M_{\odot}>10.4$ candidate members with an early-type morphology also appear to be

\footnotetext{
21 Here and in later sections, errors on fractions are calculated following Cameron (2011).
}

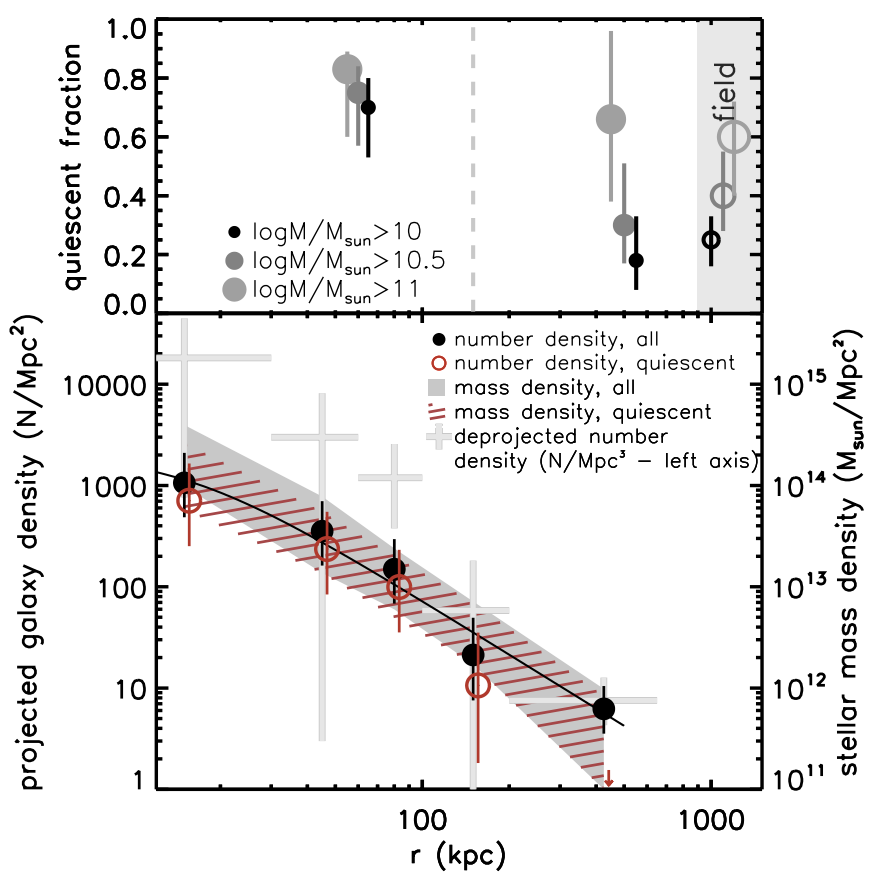

Figure 8. Bottom: number density profile of cluster galaxies as estimated from the sample of candidate members (see the text for details). Black and red symbols show the whole population more massive than $10^{10} M_{\odot}$ and the sub-sample of galaxies classified as passive, respectively (black and red symbols are slightly offset for clarity). Error bars include the Poisson error and the uncertainties in membership determination (see the text). The gray-shaded and red-hatched areas show the inferred stellar mass density profiles (right-hand scale) for the same two samples $\left(>10^{10} M_{\odot}\right.$, whole population and passive sources, respectively). The light gray crosses show a simplistic deprojection of the observed density profile (black symbols) assuming spherical symmetry (see the text; units are galaxies $/ \mathrm{Mpc}^{3}$ on the left-hand $y$-axis). Top: the fraction of passive galaxies in two radial bins of clustercentric radius $(r<150 \mathrm{kpc}$ and $150<r<700 \mathrm{kpc}$, solid symbols) as estimated from the sample of candidate cluster members (see the text for details). The passive fraction as measured from these same data in the field (galaxies classified as interlopers at $1.5<z<2.5$ ) is shown with empty symbols. Black, dark gray, and light gray symbols show mass-complete samples with $\log M / M_{\odot}>10,10.5$, and 11, respectively.

(A color version of this figure is available in the online journal.)

passive, with a similar fraction in our sample of interlopers at $1.5<z<3$, although statistics are too poor to draw conclusions. For comparison, Bell et al. (2012) find about $60 \%$ of early-type galaxies to be passive, at $z \sim 2$, down to a stellar mass limit of $5.5 \times 10^{10} M_{\odot}$.

\subsection{The Environmental Signature on Galaxy Populations}

In Figure 8, we show the projected number density profile for the whole mass-complete sample of candidate members more massive than $10^{10} M_{\odot}$ as well as for passive galaxies in this sample, and the related stellar mass profiles. For the purpose of this figure, the cluster center is taken to be the center of the galaxy overdensity, roughly coincident with a complex multi-component galaxy system with asymmetric halos and tails suggestive of ongoing merging. Gobat et al. (2011) identified this system as the possible proto brightest-cluster-galaxy (BCG) still in a very active formation phase. This object is offset by $\sim 50 \mathrm{kpc}$ (in projection) from the estimated center of the X-ray emission. Note that this offset is similar to what is observed in lower redshift clusters and groups, although in our case it is comparable to the uncertainty on the X-ray centroid position (Fassbender et al. 2011a; George et al. 2012). All profiles shown in Figure 8 take into account the contamination by interlopers 
by resampling multiple times the sample of candidate members, according to the contamination estimates discussed above. The errors shown on the number density profiles are the largest between the Poisson error on the counts and the scatter due to the resampling. For stellar mass profiles, an error of a factor two in stellar mass is included. At these masses, the impact of less-likely ("possible") members is marginal, and we verify that the inclusion or exclusion of these galaxies does not affect the profiles. The light gray crosses in Figure 8 show, as a simplistic illustration, the density profile obtained by deprojecting the observed profile (black points) assuming spherical symmetry, with a simple approach similar to McLaughlin (1999). We assume no significant contribution to the overdensity beyond $650 \mathrm{kpc}$. Based on this estimate, the average volume number density of massive galaxies $\left(>10^{10} M_{\odot}\right)$ within the region probed by this profile ( $650 \mathrm{kpc}$ from the cluster center) would be about $250 \pm 100$ times the density in the field at $z \sim$ 2 (from wide-field measurements, e.g., Muzzin et al. 2013; see also Section 2.3.1), reaching central densities 4-5 orders of magnitude larger than in the field within $100 \mathrm{kpc}$ from the cluster center. We stress that this is only a simplistic approximation for illustration purposes, and of course we have no proof - and likely no expectations - that this cluster is spherically symmetric.

The purpose of Figure 8 is to quantitatively show the increased galaxy density of candidate members in the cluster central region. A proper investigation of the shape of the galaxy number density profile is beyond the scope of this work, but we show as a reference the best-fitting projected $\beta$-model ${ }^{22}$ $\left(\Sigma(r)=\sigma_{0}\left(1+\left(r / r_{\text {core }}\right)^{2}\right)^{-\beta} ;\right.$ Cavaliere \& Fusco-Femiano 1978; with $\sigma_{0}$ the central projected density, $r_{\text {core }}$ the core radius, and $\beta$ the outer slope) to the number density profile (black points) as a black line. The profile suggests that if there is a core, it is very small (core radius $20_{-10}^{+30} \mathrm{kpc}$ ), as also observed in low-redshift clusters (e.g., Biviano \& Girardi 2003). The bestfit value of $\beta(\sim 0.9)$ is close to the typical values observed in the nearby universe (e.g., Popesso et al. 2004). Given the small offset between the X-ray centroid and the center of the overdensity, we note that this figure would be essentially the same if we adopted the X-ray centroid as the cluster center. The only relevant effect would be the increase of the core size to $\sim 50 \mathrm{kpc}$.

The top panel of Figure 8 shows the fraction of candidate members that are classified as passive in two radial bins (within and beyond a clustercentric distance of $150 \mathrm{kpc}$ ) for three masslimited samples $\left(\log M / M_{\odot}>10,10.5\right.$, and 11). In spite of the relatively poor statistics and contamination by field galaxies, this figure clearly shows that a larger fraction of galaxies has already suppressed star formation in the cluster center, corresponding to the high-density region shown by the profile in the bottom panel. The effect is seen in all the mass-limited samples shown, with the possible exception of the most massive systems $\left(>10^{11} M_{\odot}\right)$. Statistics are too poor to draw any conclusion, but the lack of a clear environmental effect for the most massive galaxies might hint at a dominant role of mass-related factors (the so-called mass quenching, e.g., Baldry et al. 2006; Peng et al. 2010b) in quenching galaxies at the highest masses, at this epoch and for this kind of environment (but see, e.g., Muzzin et al. 2012 for clusters at $z \sim 1$ ). In the outer bin (between 150 and $\sim 700 \mathrm{kpc}$ ), the passive fraction is consistent with the field value (estimated

\footnotetext{
22 A generalization of core profiles that is often used to describe cluster galaxy
} number density profiles (e.g., Girardi et al. 1998; Lemze et al. 2009). from these same data using galaxies classified as interlopers at $1.5<z<2.5$ and taking into account the resampling of candidate members). However, we remind the reader that where the overdensity of cluster galaxies drops, dilution from contamination dramatically affects how well we can recover their properties, making them appear more similar to the field sample. Statistically correcting for contamination by interlopers, as it is done here, is expected to give lower passive fractions (if field galaxies have a higher star-forming fraction), especially in the outer regions where field contamination is more significant. Finally, we note that the passive fraction that we measure in the field is consistent with previous determinations at $z \sim 2$. For instance, we find a passive fraction of $50 \% \pm 15 \%$ at masses $\log M / M_{\odot}=11.15 \pm 0.35$, close-given the uncertainties-to the estimates of, e.g., Daddi et al. (2005a), Brammer et al. (2011), and Patel et al. (2013).

Compared to $z \sim 1$ clusters, we find, as expected, higher star-forming fractions at $z \sim 2$ (however, we recall the caveat just discussed above that might bias our estimates high). For instance, in the range $10.25 \lesssim \log M / M_{\odot} \lesssim 10.95$, Muzzin et al. (2012) find about $20 \%$ of cluster galaxies with still active star formation within $200 \mathrm{kpc}$ from the cluster core, while we estimate about $50 \%$. However, at high stellar masses $\left(\log M / M_{\odot}>10.95\right), 70_{-20}^{+10} \%$ of galaxies within $200 \mathrm{kpc}$ appear to be already passive, a result that is not likely to be produced by field contamination. This percentage is already similar to the low star-forming fractions $(\sim 20 \%)$ estimated by Muzzin et al. in their $z \sim 1$ sample.

\subsection{The High-mass Tail}

At $z<1.5$, very massive galaxies are a characteristic population of cluster cores, generally exhibiting the most evolved morphological structures and stellar populations. However, studies of $z>1.5$ clusters often show significant activity (from both the star formation and mass assembly points of view), even in the high-mass tail of cluster galaxy populations (as discussed in the Introduction).

Based on the results of SED fitting, in our sample there are nine candidate members with stellar masses exceeding $10^{11} M_{\odot}$. Six of these objects are spectroscopic members. These galaxies are mostly concentrated close to the cluster center, with five of them within a clustercentric distance $d_{\mathrm{cl}} \lesssim 100 \mathrm{kpc}$. Only two are classified as actively star forming based on our criteria: one candidate at $d_{\mathrm{cl}}>600 \mathrm{kpc}$ and one component of the proto-BCG system that from recent analysis seems indeed to be associated with the cluster (Gobat et al. 2013). While the photometry of this latter source is likely significantly affected by the presence of multiple components and neighbors, a detection in the Herschel PACS imaging indeed suggests a star formation rate of order $\sim 100 M_{\odot} \mathrm{yr}^{-1}$ (Gobat et al. 2013). All other seven $>10^{11} M_{\odot}$ candidate members are classified as passive. Four of them have an early-type morphology, although one is embedded in a large asymmetric halo with features suggestive of a recent interaction. The remaining three galaxies classified as passive are (1) a likely member with disky morphology, (2) a possible member with a distorted shape showing a large tail, and (3) a galaxy very close $\left(\sim 1^{\prime \prime} .5\right)$ to the star-forming component of the proto-BCG mentioned above, identified in Gobat et al. (2011) as a proto-BCG component itself. We were not able to obtain a reliable Sersic fit for this source, likely due to its complicated surroundings. The F140W images of these three galaxies are shown in Figure 9. 

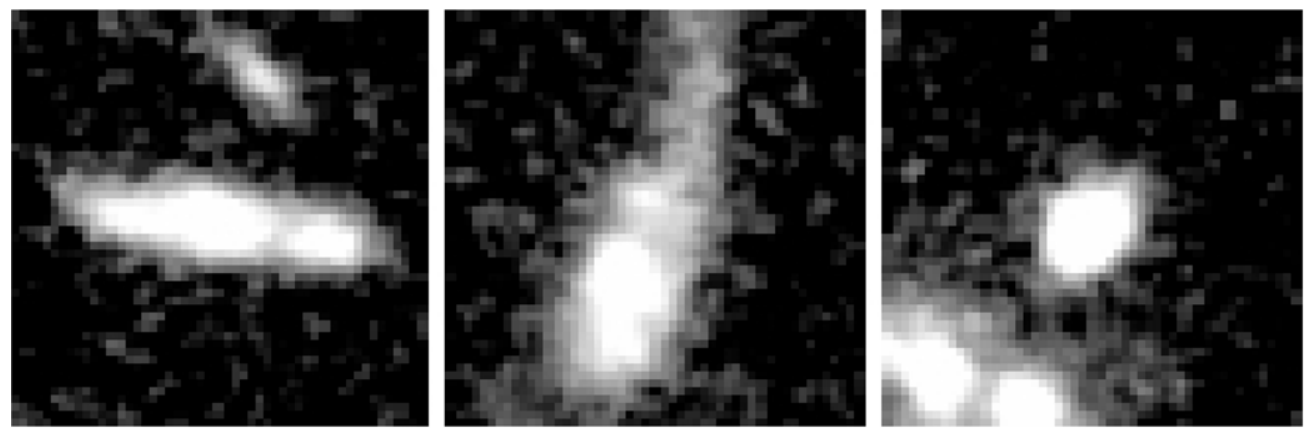

Figure 9. WFC3 F140W cutouts of the three massive passive (candidate) members that do not have an early-type morphology (left and middle panels), or lack a reliable fit (right panel). Cutout size is $3^{\prime \prime}$, or $\sim 26 \mathrm{kpc}$ at $z=2$.

In spite of the mentioned caveats, these observations would thus picture the high-mass-end galaxy population of this cluster as a mix of passive galaxies with already-established earlytype morphologies and galaxies that are instead still actively forming their stars, assembling their mass, or reshaping their structure, in some cases, through interactions. We note that, with the exception of the proto-BCG complex, the central region within $<150 \mathrm{kpc}$ of the cluster center hosts the most evolved of these very massive galaxies. Those still actively star forming or with disk or irregular morphologies typically lie outside of the cluster core, at $d_{\mathrm{cl}}>350 \mathrm{kpc}$.

As already mentioned in Section 3, the most massive galaxies in the core of this cluster already show a very high passive fraction $\left(83_{-20}^{+6} \%\right.$ for the fully spectroscopically confirmed sample of $M>10^{11} M_{\odot}$ members within $150 \mathrm{kpc}$ of the cluster center). This fraction is close to the estimate by Raichoor \& Andreon (2012) for very massive galaxies in the central area of JKCS 041, at a roughly similar redshift.

As also found in other studies at this redshift, the fraction of very massive galaxies that have already attained an earlytype morphology is significantly lower than at lower redshift. Both in our cluster and $1.5<z<2.5$ field samples, $40 \% \pm$ $15 \%$ of galaxies more massive than $10^{11} M_{\odot}$ are classified as morphological early types (or $20_{-6}^{+20} \%$ for $\gtrsim 2 \times 10^{11} M_{\odot}$, in agreement with Buitrago et al. 2013). On the other hand, the fraction of early types could be larger for $>10^{11} M_{\odot}$ passive galaxies $(60 \% \pm 15 \%$, larger than the $35 \% \pm 15 \%$ estimated by van der Wel et al. 2011, but still consistent given the significant uncertainties).

\subsection{An Estimate of the Cluster Mass from Its Host Galaxies}

As reported in Gobat et al. $(2011,2013)$, the total mass of $\mathrm{Cl} \mathrm{J} 1449+0856$ as inferred from its X-ray luminosity would be $M_{200}=5.3 \pm 110^{13} M_{\odot}$. Gobat et al. (2011) also attempted an independent estimate of the cluster mass based on the stellar mass contained in the red galaxies in the very central $\left(20^{\prime \prime}\right.$; $\sim 170 \mathrm{kpc}$ ) overdensity. We attempt here a refinement of this estimate based on the sample of candidate members within a clustercentric radius of $500 \mathrm{kpc}$. We stress that this only gives a very rough indication of the cluster mass, since besides the biases related to the selection of candidate members, which are extensively discussed above, there are many additional important uncertainties. Among these, our ignorance of the cluster virial radius and of the redshift evolution up to $z \sim 2$ of the relation between cluster total mass and stellar mass in galaxies.

Given the cluster redshift as well as the previous mass estimates, it is reasonable to assume that the cluster virial radius is likely not much larger than $\sim 500 \mathrm{kpc}$. Therefore, an $r<500 \mathrm{kpc}$ area accounts for most of the mass in galaxies in this system (as would also be suggested by Figure 8). We estimate the stellar mass in galaxies within this area, ${ }^{23}$ taking into account contamination by interlopers as discussed above, and extrapolating down to stellar masses of $10^{7} M_{\odot}$ assuming the shape of the mass function measured in the field at $1.5<z<2$ by Ilbert et al. (2010). The stellar mass calculated in this way is $2 \pm 1 \times 10^{12} M_{\odot}$. Based on this, we then estimate the cluster mass using its relation (in the nearby universe) with stellar mass in galaxies as determined by Andreon (2012). Since, as discussed above, we do not know the $r_{500}$ or $r_{200}{ }^{24}$ of this cluster, we apply both local calibrations based on stellar mass within $r_{500}$ and $r_{200}$, in the reasonable assumption that the $500 \mathrm{kpc}$ radius we use must be between or close to one of them. The two estimates, $4-5 \times 10^{13} M_{\odot}$, are consistent given the uncertainty of at least $50 \%$. This would correspond to a stellar mass fraction within the $r<500 \mathrm{kpc}$ area of $\sim 4 \%-5 \%$, also in agreement with other measurements up to $z \sim 1$ (Giodini et al. 2009; Leauthaud et al. 2012 for the same IMF). On the other hand, while there is currently no evidence for a significant evolution of the stellar mass fraction in clusters up to $z \sim 1$, there might well be a stronger evolution between $z \sim 1$ and 2. The actual amount of evolution is difficult to quantify, and we note just for reference that the Bower et al. (2006) semianalytic model predicts a slightly lower stellar mass fraction for group/cluster-sized halos at $z=2$ (Balogh et al. 2008). This lower stellar mass fraction would thus mildly increase, by $\sim 30 \%$, our estimate for the cluster mass.

In any case, our revised estimate of the cluster mass based on stellar mass in galaxies is close to previous determinations. We stress nonetheless once more that, given the significant assumptions and uncertainties involved, this remains only a crude guess.

\section{PASSIVE EARLY-TYPE GALAXIES}

According to the criteria described above, we identify eight passive "likely" members and four passive "possible" members, down to the completeness mass limit of $8.5 \times 10^{9} \mathrm{M}_{\odot}$. As discussed above and shown in Figure 4, these candidate members - and in particular those most likely associated with the cluster-tend to be located in the cluster core at a clustercentric radius of $<150 \mathrm{kpc}$. As expected, the fraction of

\footnotetext{
23 We correct for a small fraction of uncovered area beyond $300 \mathrm{kpc}$; see, e.g., Figure 5.

24 As for the usual definition, $r_{500}$ and $r_{200}$ are the radii within which the mean density of the cluster is 500 and 200 times, respectively, the critical density of the universe at the cluster redshift.
} 

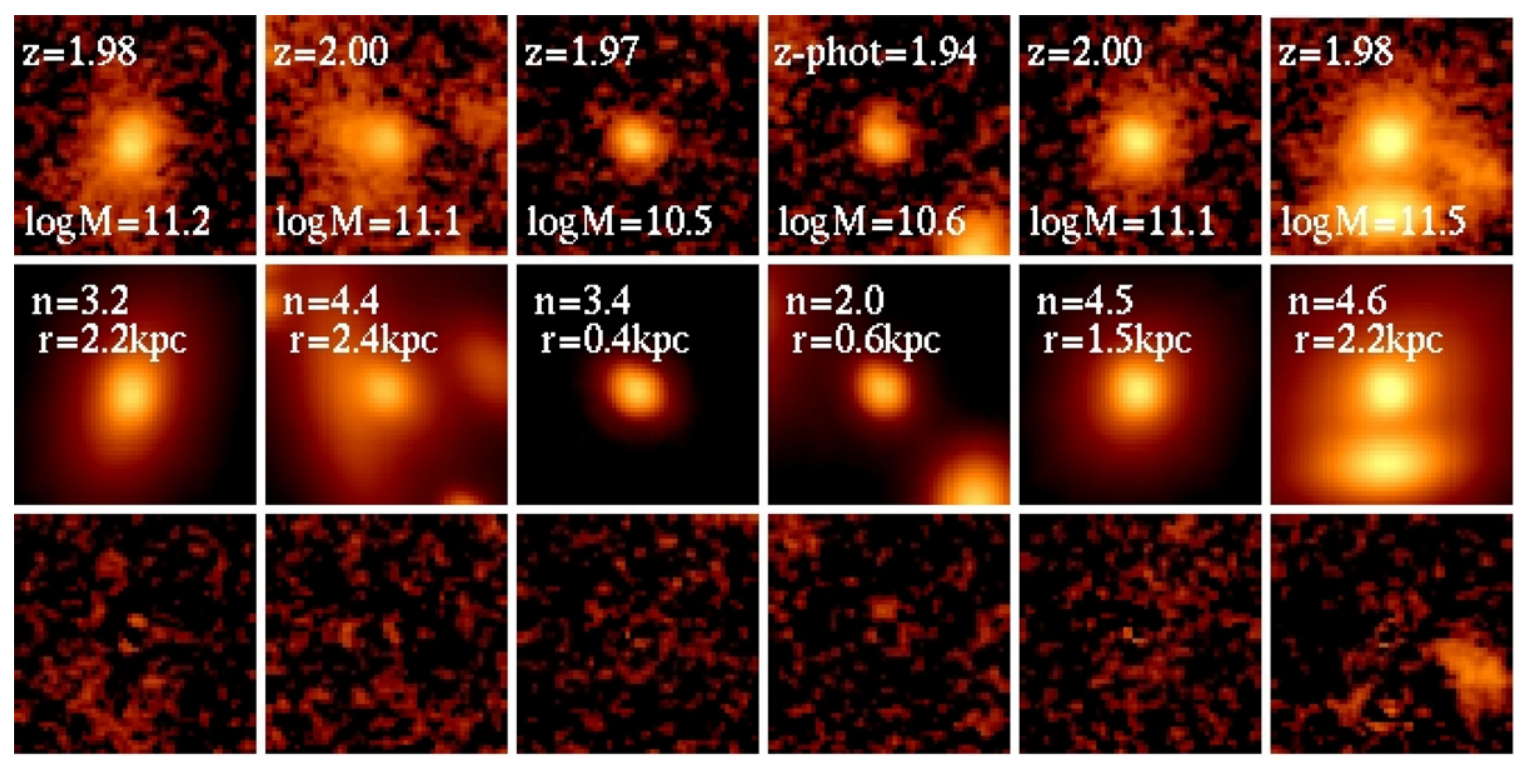

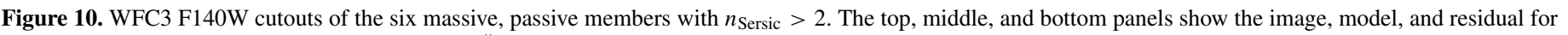
each of these sources, respectively. Cutout size is $3^{\prime \prime}$, or $\sim 26 \mathrm{kpc}$ at $z=2$.

(A color version of this figure is available in the online journal.)

candidate members classified as passive strongly depends on stellar mass. At masses below $\log M / M_{\odot}<10.5$, passive galaxies seem very rare: we have only two in our mass-complete sample, making up $15_{-5}^{+15} \%$ of the $9.9<\log M / M_{\odot}<10.5$ population of cluster candidates. Statistics are poor, and the exact number could be affected by contamination and photo- $z$ uncertainties, but there seems to be a paucity of passive candidate members at low stellar masses in our sample (see also, e.g., among others, Kodama et al. 2004; De Lucia et al. 2007; Ilbert et al. 2010; Rudnick et al. 2012, at lower to similar redshifts, and in different environments). The passive fraction increases at higher masses, reaching $30_{-10}^{+20} \%$ at $10.5<\log M / M_{\odot}<11$ and $\sim 80 \%$ at $\log M / M_{\odot}>11$ as quoted in Section 3.4.

Down to our limit for morphological analysis $\left(m_{140}<24.5\right.$, $M>2.5 \times 10^{10} M_{\odot}$ ), our passive sample contains eight "likely" and one "possible" candidate members. While the surface brightness distribution of most of these nine sources may be described with a $n>2$ Sersic profile and is suggestive overall of an early-type structure, this sample also includes the three massive $\left(>10^{11} M_{\odot}\right)$, passive systems with disk, distorted, or undetermined morphology that were discussed in Section 3.4 (Figure 9). These three galaxies are excluded from the following analysis. Cutouts of the six remaining bona fide passive cluster early types (five spectroscopic and one likely member) are shown in Figure 10, together with their Sersic models and residual maps. ${ }^{25}$

Analogously, in the redshift range close to the cluster $(1.5<$ $z<3$, corresponding to \pm 1 Gyr around $z=2$ ) we identify six (all spectroscopic) interlopers classified as passive and with a likely early-type morphology. ${ }^{26}$ The passive nature inferred from their photometry is also confirmed by their spectra (Gobat et al. 2013). Cutouts of the six passive interlopers are shown in Figure 11, together with their Sersic models and residual maps.

\footnotetext{
25 These include the early-type galaxy surrounded by a large asymmetric halo already mentioned in Section 3.4 - this source will be highlighted below where relevant.

26 We note that, although four of these interlopers have a similar redshift

$(1.86<z<1.90)$, the projected separation between any two of them is at least $200 \mathrm{kpc}$, and three out of four lie at more than $300 \mathrm{kpc}$ from the cluster center.
}

We include in this sample two sources with $1.5<n_{\text {Sersic }}<2$, which is below the $n_{\text {Sersic }}=2$ threshold adopted in this work, ${ }^{27}$ and one high- $n_{\text {Sersic }}$ source which shows signatures of interaction. ${ }^{28}$

We note that our membership criteria might produce a bias in interloper samples at $1.5<z<3$. In fact, we preferentially classify as candidate members objects in this redshift range unless they are bright enough, and possibly compact enough given our grism data, to measure their redshift and discard them as spectroscopic interlopers. This sample of field $z \sim 2$ passive galaxies might thus be, in principle, not completely representative of the $\log M / M_{\odot}>10.4$ population of passive galaxies at this redshift, including a possible bias toward compact sources. On the other hand, we also note that at the mass of these interlopers $\left(\gtrsim 10^{11} M_{\odot}\right)$, all the passive cluster early types are spectroscopically confirmed, so at least at these masses there is no uncertainty due to membership determination and we can make a meaningful, homogeneous comparison between cluster and field passive early-type galaxies in our data.

\subsection{The Mass-Size Relation of Passive Cluster Early Types}

In Figure 12, we show the ellipticity and circularized effective radius versus stellar mass for the passive (candidate) members with $n_{\text {Sersic }}>2$. All but one of these sources have $n_{\text {Sersic }}>2.5$, in fact. The six passive spectroscopic interlopers at $1.5<z<3$ are also shown, including those with $1.5<n_{\text {Sersic }}<2$ (spiral symbols).

While the average ellipticity of the cluster early types tends to be somewhat lower than those in the field, statistics are too poor to draw any significant conclusions concerning environmental dependence or redshift evolution. With this important caveat, we only note that the median ellipticity $(\sim 0.3)$ of the cluster early

\footnotetext{
27 There are no passive candidate members with $1.5<n_{\text {Sersic }}<2$.

28 This source is shown in the right-hand panels of Figure 11 and appears in Figure 12 with $\log M / M_{\odot}=11.2$ and an effective radius of $0.95 \mathrm{kpc}$. If we fit the faint component north of the source, its Sersic index and size are reduced by $30 \%-40 \%$. As Figure 12 shows, adopting the lower size estimate, or excluding this source (as well as the two $n_{\text {Sersic }}<2$ systems) from the sample would not change our conclusions.
} 

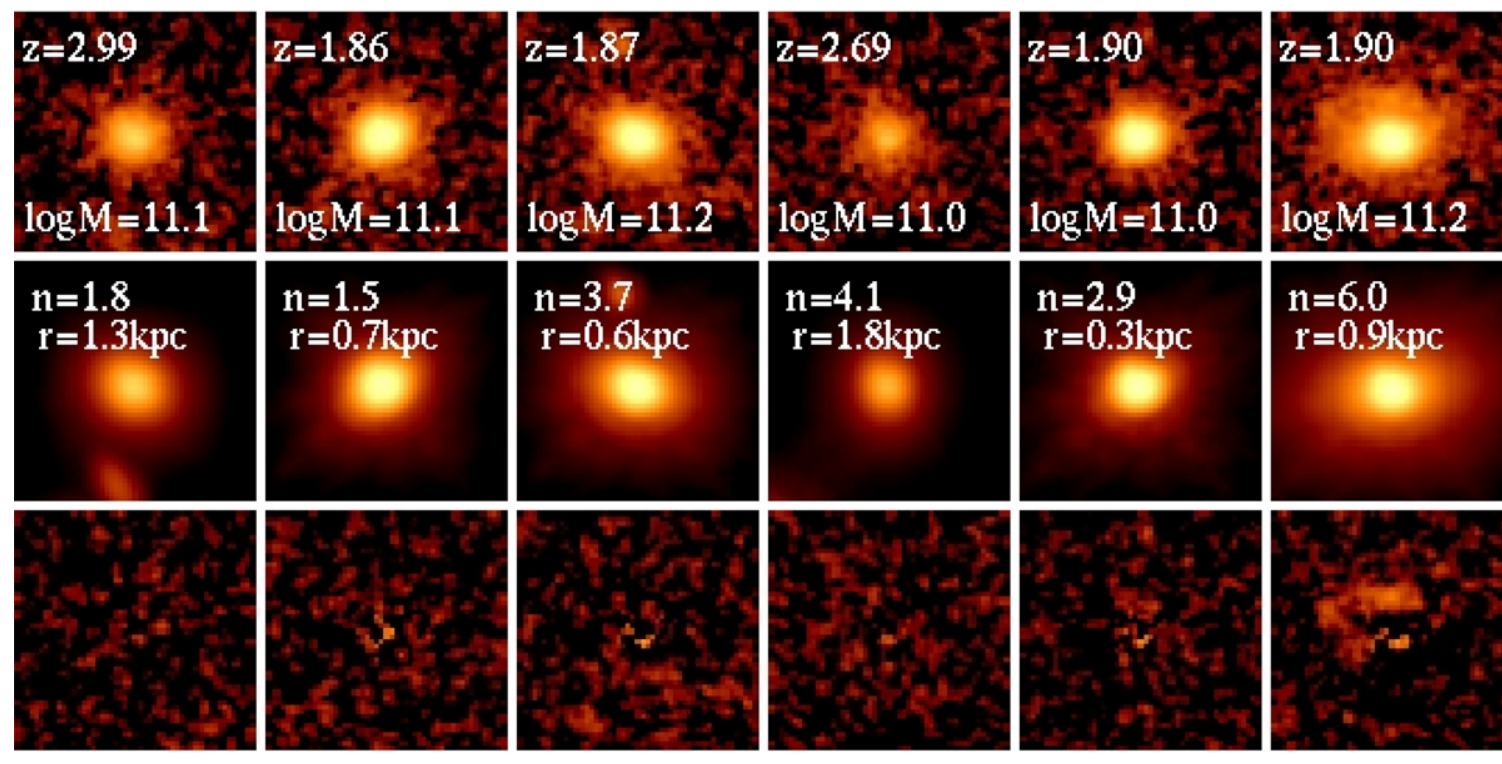

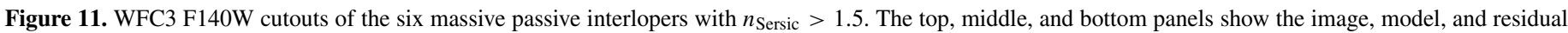
for each of these sources, respectively. Cutout size is $3^{\prime \prime}$, or $\sim 25 \mathrm{kpc}$ in the considered redshift range.

(A color version of this figure is available in the online journal.)

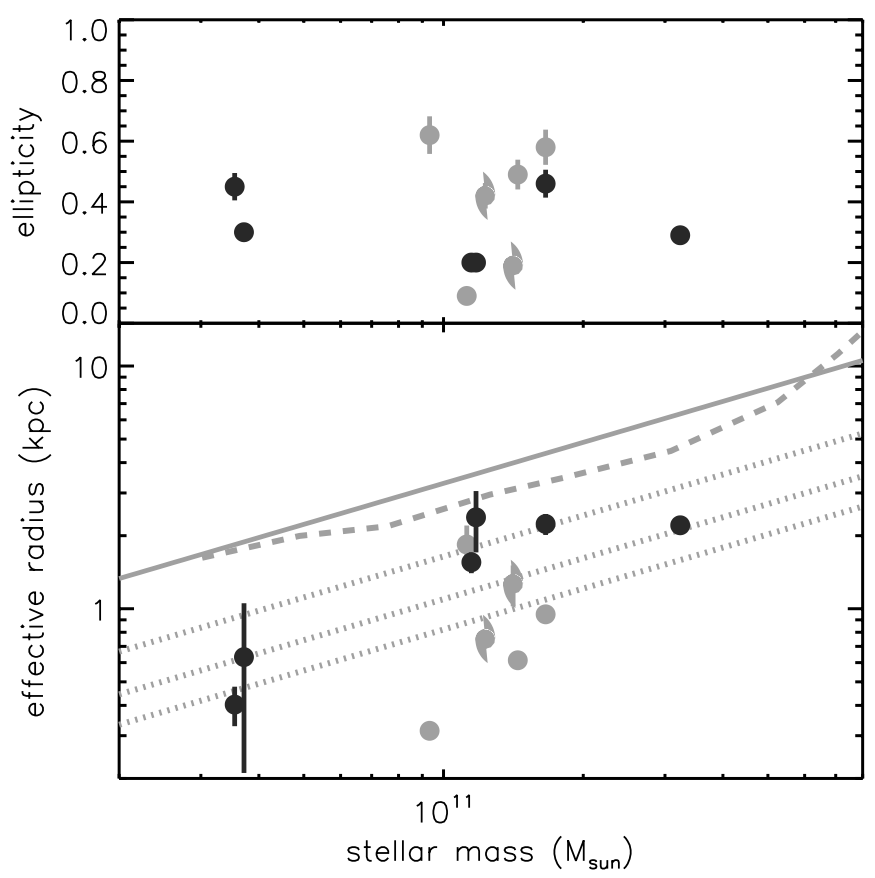

Figure 12. Ellipticity and effective radius as a function of stellar mass, for cluster (black) and field (gray, $1.5<z<3$ ) passive early-type galaxies in the probed area. Two field galaxies with $1.5<n_{\text {Sersic }}<2$ are included and are shown as spiral symbols. In the bottom panel, the solid and dashed lines show, respectively, the local determination of the stellar-mass-size relation by Shen et al. (2003) for early-type galaxies and by Valentinuzzi et al. (2010) for nearby cluster early types. The dotted lines show the Shen et al. (2003) relation scaled by factors of two, three, and four in size (top to bottom).

types seems very similar to what is observed at low redshift (e.g., Holden et al. 2009) in a similar mass range.

All galaxies in Figure 12 appear to be more compact than similarly massive early types in the nearby universe, in agreement with many previous studies at high redshift in clusters and in the field (e.g., among many others, Daddi et al. 2005b; Trujillo et al. 2006a; Zirm et al. 2007; Cimatti et al. 2008; van der Wel et al. 2008; Rettura et al. 2010; Williams et al. 2010;
Cassata et al. 2011; Cameron et al. 2011, but see also, e.g., Saracco et al. 2009; Onodera et al. 2010; Mancini et al. 2010).

For comparison, we show in Figure 12 the most commonly used local reference relation (Shen et al. 2003) and the determination by Valentinuzzi et al. (2010) for nearby cluster early types. While the Shen et al. (2003) relation has been shown to be affected by some errors and biases (e.g., Guo et al. 2009; Taylor et al. 2010; Valentinuzzi et al. 2010), due to its widespread use in previous work we use it as the $z=0$ reference to compute size evolution factors. We remind the reader that while our morphological analysis is carried out in the restframe optical ( $\sim 700 \AA)$, the Shen et al. (2003) sizes are still measured at longer wavelengths ( $z$ band). This might raise issues of morphological $k$-correction, although up to now it does not seem to be a serious concern for the kind of sources studied here (Cassata et al. 2010; Damjanov et al. 2011; Cameron et al. 2011).

While keeping in mind the small size of our sample, interlopers seem to have a larger spread in size and to be systematically more compact than candidate members of similar mass (Figure 12). ${ }^{29}$ As compared to the Shen et al. (2003) relation, cluster early types have smaller sizes on average by a factor $r_{e} / r_{e \text {, shen2003 }}=0.44 \pm 0.06(\mathrm{rms}$ range $\sim 0.2-0.7)$, while field early types have an average $r_{e} / r_{e \text {, shen2003 }}=0.22 \pm 0.06$ (rms range $\sim 0.1-0.5$ ). This would support (at least at masses $\gtrsim 10^{11} M_{\odot}$ ) recent claims of typically larger sizes of early types in high-redshift dense environments (Cooper et al. 2012; Papovich et al. 2012; Zirm et al. 2012; Tanaka et al. 2012; but see also Raichoor et al. 2012; Huertas-Company et al. 2013).

Several studies have claimed a correlation across a broad redshift range between the size of passive early types and the age of their stellar populations, with older galaxies having smaller sizes (e.g., Bernardi et al. 2010; Valentinuzzi et al. 2010; Saracco et al. 2011 and references therein, but see also results in, e.g., Cimatti et al. 2012; Onodera et al. 2012; Whitaker et al.

\footnotetext{
29 We note that this is not due to the large $1.5<z<3$ bin-in fact, the four very compact galaxies are very close to the cluster redshift at $1.8<z<1.9$, while the two at $z>2.5$ have sizes closer to the cluster members.
} 


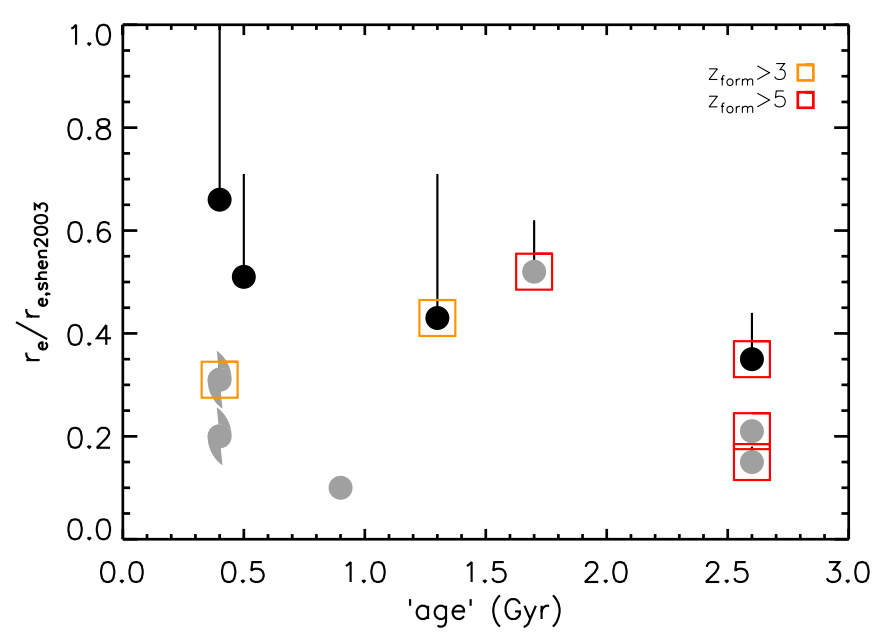

Figure 13. Size evolution factor vs. "age" for the $\gtrsim 10^{11} M_{\odot}$ cluster (black symbols) and field (gray symbols) passive early types (see Figure 12 and the text for the definition of the plotted quantities). Black lines are not errors on the size determination (see Figure 12), but show the offset in evolution factor if using M05 rather than BC03 stellar masses, for the same local reference.

(A color version of this figure is available in the online journal.)

2012). This correlation is still debated, nonetheless we note that the difference in size between cluster and field early types in Figure 12 does not seem to reflect a difference in age. We show in Figure 13 the size evolution factor for the $\gtrsim 10^{11} M_{\odot}$ sources with respect to the Shen et al. (2003) relation versus the "age," defined as the time when half of the stellar mass at the epoch of observations was formed (based on the SFH of the best-fitting model SED). At $M \gtrsim 10^{11} M_{\odot}$, the cluster and field samples are comparable in stellar mass, the galaxy sizes are relatively well constrained, and both samples are fully spectroscopically confirmed. Our poor statistics and very rough age estimates do not allow us to draw any conclusions about the age versus size relation of early types in this work, but we do not see any evidence of segregation in this figure between cluster and field galaxies (besides the larger sizes of cluster early types, already shown in Figure 12).

Assuming a size evolution of the form $(1+z)^{\alpha}, \alpha$ would be $0.75 \pm 0.15$ for cluster early types and $1.4 \pm 0.2$ for the field. This amount of size evolution for field early types at this redshift is in close agreement with previous estimates (e.g., van Dokkum et al. 2008; Buitrago et al. 2008; van der Wel et al. 2008; Cassata et al. 2011; Damjanov et al. 2011; Cimatti et al. 2012; Patel et al. 2013), although other studies have found somewhat weaker evolution (e.g., Cimatti et al. 2008; Papovich et al. 2012; but see the discussion below). We note that such a comparison may be biased by many systematics affecting the measurement of the mass-size relation, especially at different redshifts and in different data sets. Nonetheless, taken at face value, our estimate of the size evolution factor for early types is consistent with expectations from previous measurements.

Interpreting the difference in the average size of field and cluster early types as evidence that structural evolution is accelerated in the cluster environment would suggest that cluster early types reach - on average - the observed (at $z \sim 2$ ) size about 3 Gyr earlier than early types in the field. This assertion assumes a smooth evolution of the form given above in the field, down to at least $z \sim 0.8$. Note that while some work presented evidence for a smooth size evolution in the $0<z<2$ range (Damjanov et al. 2011), other studies suggest that evolution could be faster prior to $z \sim 2$ (Cimatti et al. 2012). With this important caveat, we note for comparison that a difference in stellar populations has sometimes been interpreted as a delay in the formation of the bulk of the stars in the field relative to cluster early types ranging from $\sim 0.4$ to 2 Gyr (e.g., Thomas et al. 2005; Bernardi et al. 2005; Clemens et al. 2006; van Dokkum \& van der Marel 2007; Rogers et al. 2010, all in the nearby universe). However, several other studies, including work at higher redshifts, generally concluded that if there is a delay it is small ( $\sim 0.5 \mathrm{Gyr})$, and often suggested more complex differences in the SFHs in different environments rather than an overall delay in the bulk of the star formation (e.g., Moran et al. 2005; Gobat et al. 2008; Thomas et al. 2010; Rettura et al. 2011 and references therein).

In any case, Figure 12 excludes the presence of extremely compact passive early types in the cluster, at least in the mass range and area probed here. We recall, however, that this sample does not include the passive component of the proto-BCG, for which we do not have a reliable estimate of morphological parameters, as well as the red, compact AGN host that we discarded from the spectroscopic member sample due to severe uncertainties in the determination of its properties, as discussed in Section 2.3. In principle, either or both might be examples of very compact early types in the cluster core. Besides this caveat, cluster passive early types seem to have sizes typically a factor $\sim 2-3$ smaller than similarly massive early types in the nearby universe. With the possible exception of the massive source with an asymmetric halo which, as mentioned above, may suggest a post-interaction stage, there are essentially no passive early types within $1 \sigma$ of the local relation. This might be linked to the still incomplete evolution of a massive, core galaxy population at this epoch, at least in this cluster.

On the other hand, the evolution of the mass-size relation does not necessarily imply an evolution in size of individual galaxies, and its interpretation is complicated by several biases and selection effects as discussed in many studies including, e.g., Franx et al. (2008), Bernardi et al. (2010), Hopkins et al. (2010), Williams et al. (2010), Saracco et al. (2009, 2010, 2011), and Poggianti et al. (2013). In particular, the mismatch between samples of early types at different redshifts is often considered to contribute significantly to the observed evolution of the mass-size relation, as recently summarized by, e.g., Carollo et al. (2013) and Cassata et al. (2013) with representative earlytype samples up to $z \sim 1$ and $z \sim 3$. In fact, as observed in the general field and, albeit with some differences, in all environments, continuous quenching of star-forming galaxies through cosmic times significantly increases the number density of passive galaxies - by about an order of magnitude in the field between $z \sim 2$ and today, in the mass range of our passive sample (e.g., Ilbert et al. 2013; Muzzin et al. 2013). If galaxies quenched more recently have typically larger sizes (as for the age-size correlation discussed above), the observed mass-size relation evolves even if individual early types in the high-redshift samples do not. Indeed, Valentinuzzi et al. (2010) showed that early types with sizes a factor of 2-3 below the Shen et al. (2003) relation can be found also in nearby clusters, even at high stellar masses $\left(>10^{11} M_{\odot}\right)$. Such compact galaxies tend to have older stellar populations than averagesized ones, and thus made it into the early-type samples at earlier times, shifting the average mass-size relation at higher redshifts to lower sizes. Attempts to model the effect of such a kind of progenitor bias on the mass-size relation evolution (van der Wel et al. 2009; Valentinuzzi et al. 2010) suggest that, by comparing early-type samples at $z \sim 2$ and $z \sim 0$, the 
observed $z=2$ mass-size relation could be shifted to lower sizes by a factor $\sim 30 \%$ even without any size evolution of the individual $z=2$ galaxies. On the other hand, we recall that the analysis of size evolution in age-controlled samples by Cimatti et al. (2012), albeit affected by some caveats, ${ }^{30}$ might suggest that the effect of this bias could instead be relatively mild. Although such an amount of progenitor bias would be, in any case, insufficient to fully explain our observed size evolution, it still complicates the quantification of the relevance of size evolution for individual galaxies, especially when coupled to other biases and systematics in the determination of sizes (e.g., Pannella et al. 2009; Mancini et al. 2010) and stellar masses (IMF, stellar population models, etc.). In this respect, we note that stellar masses estimated with the M05 models for the sample of early types in Figure 12 are lower by, on average, about a factor of two, thus decreasing the average evolution factor $\left(r_{e} / r_{e, \text { shen2003 }} \sim 0.7\right.$ rather than $\sim 0.4$ with BC03 masses; see also Figure 13).

\section{SUMMARY}

We have studied galaxy populations in the field of the

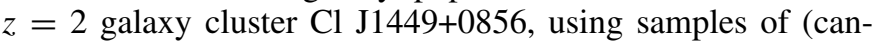
didate) members selected using spectroscopic and photometric redshifts. Our mass completeness limit is about $10^{10} M_{\odot}$ (or $2.5 \times 10^{10} M_{\odot}$ when morphological analysis is involved) at the cluster redshift. We therefore probe the population of massive cluster galaxies.

We summarize our main results as follows.

1. In spite of the residual contamination from field galaxies, which is expected to be relevant especially at low masses, the cluster clearly stands out as an overdensity both in the redshift distribution, and in the projected distribution of galaxies in the sky close to the center of the extended $\mathrm{X}$-ray emission. In the central $r<100 \mathrm{kpc}$ region, the projected number density of cluster galaxies more massive than $\sim 10^{10} M_{\odot}$ is estimated to exceed 100 galaxies $\mathrm{Mpc}^{-2}$, for a stellar mass density exceeding $10^{13} \mathrm{M}_{\odot} \mathrm{Mpc}^{-2}$.

2. The highest density cluster core is already traced by a population of massive, quiescent, early-type galaxies. On the other hand, massive star-forming galaxies, often significantly dust reddened, also populate the cluster core, as observed in other $z>1.5$ clusters. It thus appears that the core of $\mathrm{Cl} \mathrm{J} 1449+0856$ might be in a transitional phase, where a population of already massive and passive early types coexists with galaxies still actively forming their stars, and in some cases, reshaping their structure through interactions or merging.

3. Besides the central overdensity that hosts the most massive and evolved galaxy populations, a secondary galaxy concentration at $\sim 250 \mathrm{kpc}$ seems to host galaxies of a clearly distinct nature. These objects have lower masses, ongoing star formation, and late-type morphologies.

4. Environmental signatures on galaxy populations are evident within $\sim 200 \mathrm{kpc}$ from the cluster center, where the great majority of morphological early types and passive galaxies are concentrated, resulting in a clear increase in the passive fraction of massive galaxies. However, at the highest masses ( $>10^{11} M_{\odot}$ ), the passive fraction is closer to (and consistent with, given our uncertainties) the field level, which might

\footnotetext{
30 Besides the intrinsic difficulties in estimating galaxy ages, Cimatti et al. (2012) used a compilation of literature data. Therefore, age measurements were not uniform across their sample-see the original paper for details.
}

suggest a predominant role of mass over environment quenching for most massive galaxies at this redshift and in this kind of environment.

5. A first rough analysis of the spatial distribution of galaxies around the cluster center suggests a profile shape overall similar to what is observed in nearby clusters, consistent with a small-core $\beta$ model with $\beta \sim 0.9$.

6 . From the estimated stellar mass in galaxies, using the relation between stellar mass and the total mass of groups and clusters in the nearby universe, we obtain an indicative estimate for the cluster mass of about $5 \times 10^{13} M_{\odot}$, consistent with the mass inferred from the X-ray emission.

7. We observe a clear correlation between an early-type morphology and passive stellar populations, as is seen up to these redshifts also in lower density environments.

8. Massive, passive early types in this cluster are smaller on average by a factor $2-3$ with respect to the Shen et al. (2003) determination of the $z=0$ stellar mass versus size relation. However, these same objects seem to be typically larger by about a factor two than similarly-massive field galaxies at the same epoch. While statistics are still very limited, if confirmed, this finding would lend support to recent claims of accelerated structural evolution in high-redshift overdense environments.

From this study, $\mathrm{Cl} 1449+0856$ appears to be a still forming cluster that retains some expected characteristics of low-mass systems at early times, including massive galaxies still actively forming close to its center and likely infalling substructures, accreting onto the central regions lower-mass, less-evolved galaxies. On the other hand, $\mathrm{Cl} 1449+0856$ shows how, at the same time, early formed massive galaxies, quite evolved both in their structure and in their stellar content, are a major component of galaxy populations in cluster cores already 10 billion years ago.

We thank Maurilio Pannella, Anna Cibinel, Mark Sargent, Matthieu Béthermin, Gabriella De Lucia, and Stefano Andreon for their useful inputs, suggestions or comments at various stages of this work. V.S., R.G., and E.D. were supported by grants ERC-StG UPGAL 240039 and ANR-08-JCJC-0008. A.C. acknowledges the grants ASI n.I/023/12/0 "Attività relative alla fase B2/C per la missione Euclid" and MIUR PRIN 2010-2011 "The Dark Universe and the Cosmic Evolution of Baryons: from Current Surveys to Euclid." Partly based on data collected at the Subaru Telescope, operated by the National Astronomical Observatory of Japan, and at the Very Large Telescope, operated by the European Southern Observatory. Partly based on observations made under program GO-11648 with the NASA/ESA Hubble Space Telescope, which is operated by the Association of Universities for Research in Astronomy, Inc., under NASA contract NAS 5-26555. Partly based on observations made under programs GTO-64 and GO-80103 with the Spitzer Space Telescope, which is operated by the Jet Propulsion Laboratory, California Institute of Technology under a contract with NASA.

\section{REFERENCES}

Andreon, S. 2008, MNRAS, 386, 1045

Andreon, S. 2012, A\&A, 548, A83

Baldry, I. K., Balogh, M. L., Bower, R. G., et al. 2006, MNRAS, 373, 469

Balogh, M. L., McCarthy, I. G., Bower, R. G., \& Eke, V. R. 2008, MNRAS, 385, 1003

Bell, E. F., van der Wel, A., Papovich, C., et al. 2012, ApJ, 753, 167 
Bernardi, M., Shankar, F., Hyde, J. B., et al. 2010, MNRAS, 404, 2087 Bernardi, M., Sheth, R. K., Nichol, R. C., Schneider, D. P., \& Brinkmann, J. 2005, AJ, 129, 61

Bertin, E., \& Arnouts, S. 1996, A\&AS, 117, 393

Biviano, A., \& Girardi, M. 2003, ApJ, 585, 205

Bower, R. G., Benson, A. J., Malbon, R., et al. 2006, MNRAS, 370, 645

Brainerd, T. G., Blandford, R. D., \& Smail, I. 1996, ApJ, 466, 623

Brammer, G. B., van Dokkum, P. G., \& Coppi, P. 2008, ApJ, 686, 1503

Brammer, G. B., Whitaker, K. E., van Dokkum, P. G., et al. 2011, ApJ, 739,24

Bruzual, G., \& Charlot, S. 2003, MNRAS, 344, 1000

Buitrago, F., Trujillo, I., Conselice, C. J., \& Haeussler, B. 2013, MNRAS, 428, 1460

Buitrago, F., Trujillo, I., Conselice, C. J., et al. 2008, ApJL, 687, L61

Calzetti, D., Armus, L., Bohlin, R. C., et al. 2000, ApJ, 533, 682

Cameron, E. 2011, PASA, 28, 128

Cameron, E., Carollo, C. M., Oesch, P. A., et al. 2011, ApJ, 743, 146

Capak, P., Aussel, H., Ajiki, M., et al. 2007, ApJS, 172, 99

Carollo, C. M., Bschorr, T. J., Renzini, A., et al. 2013, arXiv:1302.5115

Cassata, P., Giavalisco, M., Guo, Y., et al. 2010, ApJL, 714, L79

Cassata, P., Giavalisco, M., Guo, Y., et al. 2011, ApJ, 743, 96

Cassata, P., Giavalisco, M., Williams, C. C., et al. 2013, arXix:1303.2689

Cavaliere, A., \& Fusco-Femiano, R. 1978, A\&A, 70, 677

Cimatti, A., Cassata, P., Pozzetti, L., et al. 2008, A\&A, 482, 21

Cimatti, A., Nipoti, C., \& Cassata, P. 2012, MNRAS, 422, L62

Clemens, M. S., Bressan, A., Nikolic, B., et al. 2006, MNRAS, 370, 702

Cooper, M. C., Griffith, R. L., Newman, J. A., et al. 2012, MNRAS, 419, 3018

Daddi, E., Cimatti, A., Renzini, A., et al. 2004, ApJ, 617, 746

Daddi, E., Dickinson, M., Chary, R., et al. 2005a, ApJL, 631, L13

Daddi, E., Renzini, A., Pirzkal, N., et al. 2005b, ApJ, 626, 680

Damjanov, I., Abraham, R. G., Glazebrook, K., et al. 2011, ApJL, 739, L44

De Lucia, G., Poggianti, B. M., Aragón-Salamanca, A., et al. 2007, MNRAS, 374, 809

De Lucia, G., Springel, V., White, S. D. M., Croton, D., \& Kauffmann, G. 2006, MNRAS, 366, 499

De Propris, R., Stanford, S. A., Eisenhardt, P. R., Holden, B. P., \& Rosati, P. 2007, AJ, 133, 2209

Dressler, A. 1980, ApJ, 236, 351

Eisenhardt, P. R. M., Brodwin, M., Gonzalez, A. H., et al. 2008, ApJ, 684, 905

Fassbender, R., Böhringer, H., Nastasi, A., et al. 2011a, NJPh, 13, 125014

Fassbender, R., Nastasi, A., Böhringer, H., et al. 2011b, A\&A, 527, L10

Franx, M., van Dokkum, P. G., Schreiber, N. M. F., et al. 2008, ApJ, 688, 770

George, M. R., Leauthaud, A., Bundy, K., et al. 2012, ApJ, 757, 2

Giodini, S., Pierini, D., Finoguenov, A., et al. 2009, ApJ, 703, 982

Girardi, M., Giuricin, G., Mardirossian, F., Mezzetti, M., \& Boschin, W. 1998, ApJ, 505, 74

Gobat, R., Daddi, E., Onodera, M., et al. 2011, A\&A, 526, A133

Gobat, R., Rosati, P., Strazzullo, V., et al. 2008, A\&A, 488, 853

Gobat, R., Strazzullo, V., Daddi, E., et al. 2013, arXiv:1305.3576

Guo, Y., McIntosh, D. H., Mo, H. J., et al. 2009, MNRAS, 398, 1129

Hatch, N. A., Kurk, J. D., Pentericci, L., et al. 2011, MNRAS, 415, 2993

Hayashi, M., Kodama, T., Koyama, Y., Tadaki, K.-I., \& Tanaka, I. 2011, MNRAS, 415, 2670

Hayashi, M., Kodama, T., Koyama, Y., et al. 2010, MNRAS, 402, 1980

Hilton, M., Lloyd-Davies, E., Stanford, S. A., et al. 2010, ApJ, 718, 133

Hoaglin, D. C., Mosteller, F., \& Tukey, J. W. (ed.) 1983, Understanding Robust and Exploratory Data Analysis (Wiley Series in Probability and Mathematical Statistics; New York: Wiley)

Holden, B. P., Franx, M., Illingworth, G. D., et al. 2009, ApJ, 693, 617

Hopkins, P. F., Bundy, K., Hernquist, L., Wuyts, S., \& Cox, T. J. 2010, MNRAS, 401, 1099

Huertas-Company, M., Mei, S., Shankar, F., et al. 2013, MNRAS, 428, 1715

Ilbert, O., Capak, P., Salvato, M., et al. 2009, ApJ, 690, 1236

Ilbert, O., McCracken, H. J., Le Fevre, O., et al. 2013, arXiv:1301.3157

Ilbert, O., Salvato, M., Le Floc'h, E., et al. 2010, ApJ, 709, 644

Johansson, P. H., Naab, T., \& Ostriker, J. P. 2012, ApJ, 754, 115

Kodama, T., Tanaka, I., Kajisawa, M., et al. 2007, MNRAS, 377, 1717

Kodama, T., Yamada, T., Akiyama, M., et al. 2004, MNRAS, 350, 1005

Kriek, M., van Dokkum, P. G., Labbé, I., et al. 2009, ApJ, 700, 221

Kurk, J., Cimatti, A., Zamorani, G., et al. 2009, A\&A, 504, 331

Leauthaud, A., George, M. R., Behroozi, P. S., et al. 2012, ApJ, 746, 95

Lemze, D., Broadhurst, T., Rephaeli, Y., Barkana, R., \& Umetsu, K. 2009, ApJ, 701,1336

Lidman, C., Rosati, P., Tanaka, M., et al. 2008, A\&A, 489, 981

Mancini, C., Daddi, E., Renzini, A., et al. 2010, MNRAS, 401, 933
Mancone, C. L., Gonzalez, A. H., Brodwin, M., et al. 2010, ApJ, 720, 284 Maraston, C. 2005, MNRAS, 362, 799

Maraston, C., Pforr, J., Renzini, A., et al. 2010, MNRAS, 407, 830

McLaughlin, D. E. 1999, AJ, 117, 2398

Mei, S., Holden, B. P., Blakeslee, J. P., et al. 2009, ApJ, 690, 42

Mei, S., Stanford, S. A., Holden, B. P., et al. 2012, ApJ, 754, 141

Moran, S. M., Ellis, R. S., Treu, T., et al. 2005, ApJ, 634, 977

Muzzin, A., Marchesini, D., Stefanon, M., et al. 2013, arXiv:1303.4409

Muzzin, A., Wilson, G., Yee, H. K. C., et al. 2012, ApJ, 746, 188

Onodera, M., Daddi, E., Gobat, R., et al. 2010, ApJL, 715, L6

Onodera, M., Renzini, A., Carollo, M., et al. 2012, ApJ, 755, 26

Pannella, M., Gabasch, A., Goranova, Y., et al. 2009, ApJ, 701, 787

Papovich, C. 2008, ApJ, 676, 206

Papovich, C., Bassett, R., Lotz, J. M., et al. 2012, ApJ, 750, 93

Papovich, C., Finkelstein, S. L., Ferguson, H. C., Lotz, J. M., \& Giavalisco, M. 2011, MNRAS, 412, 1123

Papovich, C., Momcheva, I., Willmer, C. N. A., et al. 2010, ApJ, 716, 1503

Patel, S. G., Holden, B. P., Kelson, D. D., et al. 2012, ApJL, 748, L27

Patel, S. G., Kelson, D. D., Holden, B. P., et al. 2009, ApJ, 694, 1349

Patel, S. G., van Dokkum, P. G., Franx, M., et al. 2013, ApJ, 766, 15

Peng, C. Y., Ho, L. C., Impey, C. D., \& Rix, H.-W. 2002, AJ, 124, 266

Peng, C. Y., Ho, L. C., Impey, C. D., \& Rix, H.-W. 2010a, AJ, 139, 2097

Peng, Y.-j., Lilly, S. J., Kovač, K., et al. 2010b, ApJ, 721, 193

Poggianti, B., Calvi, R., Bindoni, D., et al. 2013, ApJ, 762, 77

Popesso, P., Böhringer, H., Brinkmann, J., Voges, W., \& York, D. G. 2004, A\&A, 423, 449

Postman, M., Franx, M., Cross, N. J. G., et al. 2005, ApJ, 623, 721

Raichoor, A., \& Andreon, S. 2012, A\&A, 537, A88

Raichoor, A., Mei, S., Stanford, S. A., et al. 2012, ApJ, 745, 130

Rettura, A., Mei, S., Stanford, S. A., et al. 2011, ApJ, 732, 94

Rettura, A., Rosati, P., Nonino, M., et al. 2010, ApJ, 709, 512

Rogers, B., Ferreras, I., Pasquali, A., et al. 2010, MNRAS, 405, 329

Rosati, P., Tozzi, P., Gobat, R., et al. 2009, A\&A, 508, 583

Rudnick, G. H., Tran, K.-V., Papovich, C., Momcheva, I., \& Willmer, C. 2012, ApJ, 755, 14

Salpeter, E. E. 1955, ApJ, 121, 161

Santos, J. S., Fassbender, R., Nastasi, A., et al. 2011, A\&A, 531, L15

Saracco, P., Longhetti, M., \& Andreon, S. 2009, MNRAS, 392, 718

Saracco, P., Longhetti, M., \& Gargiulo, A. 2010, MNRAS, 408, L21

Saracco, P., Longhetti, M., \& Gargiulo, A. 2011, MNRAS, 412, 2707

Sargent, M. T., Carollo, C. M., Lilly, S. J., et al. 2007, ApJS, 172, 434

Shen, S., Mo, H. J., White, S. D. M., et al. 2003, MNRAS, 343, 978

Spitler, L. R., Labbé, I., Glazebrook, K., et al. 2012, ApJL, 748, L21

Steidel, C. C., Adelberger, K. L., Shapley, A. E., et al. 2005, ApJ, 626, 44

Strazzullo, V., Rosati, P., Pannella, M., et al. 2010, A\&A, 524, A17

Tanaka, M., De Breuck, C., Venemans, B., \& Kurk, J. 2010a, A\&A, 518, A18

Tanaka, M., Finoguenov, A., Mirkazemi, M., et al. 2013, PASJ, 65, 17

Tanaka, M., Finoguenov, A., \& Ueda, Y. 2010b, ApJL, 716, L152

Taylor, E. N., Franx, M., Glazebrook, K., et al. 2010, ApJ, 720, 723

Thomas, D., Maraston, C., Bender, R., \& de Oliveira, C. M. 2005, ApJ, 621,673

Thomas, D., Maraston, C., Schawinski, K., Sarzi, M., \& Silk, J. 2010, MNRAS, 404, 1775

Tran, K., Papovich, C., Saintonge, A., et al. 2010, ApJL, 719, L126

Trujillo, I., Feulner, G., Goranova, Y., et al. 2006a, MNRAS, 373, L36

Trujillo, I., Förster Schreiber, N. M., Rudnick, G., et al. 2006b, ApJ, 650, 18

Valentinuzzi, T., Fritz, J., Poggianti, B. M., et al. 2010, ApJ, 712, 226

van der Wel, A., Bell, E. F., van den Bosch, F. C., Gallazzi, A., \& Rix, H. 2009, ApJ, 698, 1232

van der Wel, A., Holden, B. P., Franx, M., et al. 2007, ApJ, 670, 206

van der Wel, A., Holden, B. P., Zirm, A. W., et al. 2008, ApJ, 688, 48

van der Wel, A., Rix, H.-W., Wuyts, S., et al. 2011, ApJ, 730, 38

van Dokkum, P. G., Franx, M., Kriek, M., et al. 2008, ApJL, 677, L5

van Dokkum, P. G., \& van der Marel, R. P. 2007, ApJ, 655, 30

Wetzel, A. R., Tinker, J. L., \& Conroy, C. 2012, MNRAS, 424, 232

Whitaker, K. E., Kriek, M., van Dokkum, P. G., et al. 2012, ApJ, 745, 179

Whitaker, K. E., Labbé, I., van Dokkum, P. G., et al. 2011, ApJ, 735, 86

Williams, R. J., Quadri, R. F., Franx, M., van Dokkum, P., \& Labbé, I. 2009, ApJ, 691, 1879

Williams, R. J., Quadri, R. F., Franx, M., et al. 2010, ApJ, 713, 738

Wuyts, S., Förster Schreiber, N. M., van der Wel, A., et al. 2011, ApJ, 742,96

Wuyts, S., Labbé, I., Franx, M., et al. 2007, ApJ, 655, 51

Zirm, A. W., Toft, S., \& Tanaka, M. 2012, ApJ, 744, 181

Zirm, A. W., van der Wel, A., Franx, M., et al. 2007, ApJ, 656, 66 